INSTITUT NATIONAL DE RECHERCHE EN INFORMATIQUE ET EN AUTOMATIQUE

\title{
Locally implicit discontinuous Galerkin method for time domain electromagnetics
}

\author{
Victorita Dolean - Hassan Fahs — Loula Fezoui — Stéphane Lanteri
}

\section{$\mathbf{N}^{\circ} 6990$}

Janvier 2007

Thème NUM

\section{apport}

derecherche 


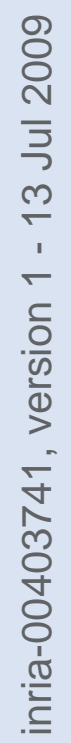




\title{
Locally implicit discontinuous Galerkin method for time domain electromagnetics
}

\author{
Victorita Dolean $^{*}$, Hassan Fahs , Loula Fezoui , Stéphane Lanteri ${ }^{\dagger}$ \\ Thème NUM — Systèmes numériques \\ Projet Nachos
}

Rapport de recherche $\mathrm{n}^{\circ} 6990$ - Janvier 2007 - 37 pages

\begin{abstract}
In the recent years, there has been an increasing interest in discontinuous Galerkin time domain (DGTD) methods for the solution of the unsteady Maxwell equations modeling electromagnetic wave propagation. One of the main features of DGTD methods is their ability to deal with unstructured meshes which are particularly well suited to the discretization of the geometrical details and heterogeneous media that characterize realistic propagation problems. Such DGTD methods most often rely on explicit time integration schemes and lead to block diagonal mass matrices. However, explicit DGTD methods are also constrained by a stability condition that can be very restrictive on highly refined meshes and when the local approximation relies on high order polynomial interpolation. An implicit time integration scheme is a natural way to obtain a time domain method which is unconditionally stable but at the expense of the inversion of a global linear system at each time step. A more viable approach consists in applying an implicit time integration scheme locally in the refined regions of the mesh while preserving an explicit time scheme in the complementary part, resulting in an hybrid explicit-implicit (or locally implicit) time integration strategy. In this paper, we report on our recent efforts towards the development of such a hybrid explicit-implicit DGTD method for solving the time domain Maxwell equations on unstructured simplicial meshes. Numerical experiments for 2D and 3D propagation problems in homogeneous and heterogeneous media illustrate the possibilities of the method for simulations involving locally refined meshes.
\end{abstract}

Key-words: computational electromagnetics, time domain Maxwell's equations, discontinuous Galerkin method, unstructured tetrahedral meshes, hybrid explicit-implicit scheme.

\footnotetext{
* University of Nice-Sophia Antipolis, J.A. Dieudonné Mathematics Laboratory, CNRS UMR 6621, F06108 Nice Cedex, France

$\dagger$ INRIA Sophia Antipolis, Nachos project-team, 2004 Route des Lucioles, BP 93, F-06902 Sophia Antipolis Cedex, France
}

Unité de recherche INRIA Sophia Antipolis

2004, route des Lucioles, BP 93, 06902 Sophia Antipolis Cedex (France)

Téléphone : +334923877 77 - Télécopie : +33492387765 


\section{Méthode Galerkin discontinue localement implicite pour l'électromagnétisme en domaine temporel}

Résumé : Ces dernières années, un intérêt croissant a été porté aux méthodes de type Galerkin discontinu utilisant des maillages non-structurés ces derniers étant particulièrement bien adaptés à la discrétisation des détails géométriques qui caractérisent les applications réalistes. Les méthodes de type Galerkin discontinu en domaine temporel font le plus souvent appel à des schémas d'intégration en temps explicites et sont soumises à des limites de stabilité qui s'avèrent contraignantes en maillages localement raffinés ou lorsque l'ordre d'interpolation local est élevé. Un schéma d'intégration implicite est une voie naturelle pour aboutir à une méthode numérique inconditionnellement stable. Cependant, un tel schéma conduit à la résolution d'un système linéaire global à chaque pas de temps effaçant du même coup un des principaux avantages des formulations de type Galerkin discontinu. Une approche plus viable consiste à appliquer un schéma implicite localement dans les zones du maillages qui sont raffinées, et à préserver l'utilisation d'un schéma explicite pour les équations associées aux éléments situés dans la partie complémentaire du maillage. On obtient ainsi un schéma d'intégration en temps hybride explicite-implicite (ou localement implicite). Dans ce rapport, nous étudions une méthode DGTD hybride explicite-implicite de ce type pour la résolution numérique du système des équations de Maxwell en domaine temporel sur des maillages non-structurés en simplexes. Des expériences numériques en $2 \mathrm{D}$ et $3 \mathrm{D}$ portant sur des problèmes de propagation en milieux homogènes et hétérogènes permettent d'illustrer les possibilités d'une telle méthode pour des simulations mettant en jeu des maillages localement raffinés.

Mots-clés : électromagnétisme numérique, équations de Maxwell en domaine temporel, méthodes Galerkin discontinues, schéma hybride explicite-implicite. 


\section{Contents}

1 Introduction $\quad 4$

2 Continuous problem $\quad 6$

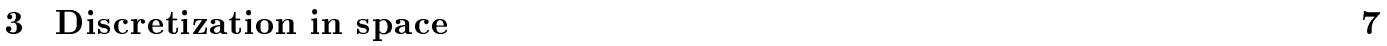

4 Time discretization $\quad 9$

4.1 Explicit time scheme . . . . . . . . . . . . . . . . . . . . . 10

4.2 Implicit time scheme . . . . . . . . . . . . . . . . . . . . 11

4.3 Hybrid explicit-implicit time scheme . . . . . . . . . . . . . . . . 11

$5 \quad$ Stability study of the hybrid explicit-implicit DGTD- $\mathbb{P}_{p}$ method 13

6 Numerical assessment of accuracy in 2D 18

6.1 Propagation of an eigenmode in a perfectly conducting cavity . . . . . . . . . 19

6.2 Scattering of a plane wave by a dielectric cylinder . . . . . . . . . . . . . . . 19

7 Numerical and performance results in 3D 22

7.1 Scattering of a plane wave by an aircraft . . . . . . . . . . . . . . . . 26

7.2 Exposure of head tissues to a localized source radiation . . . . . . . . . . 28

8 Conclusion $\quad 34$ 


\section{Introduction}

Nowadays, a variety of methods exist for the numerical treatment of the time domain Maxwell equations, ranging from the well established and still prominent finite difference time domain (FDTD) methods based on Yee's scheme [Yee66]-[TH05] to the more recent finite element time domain (FETD) and discontinuous Galerkin time domain (DGTD) methods [PFC06]-[HW02]-[CCR05]-[FLLP05]-[MR05]-[CFP06]. The use of unstructured meshes (based on quadrangles or triangles in two space dimensions, and hexahedra or tetrahedra in three space dimensions) is an intrinsic feature of the latter methods which can thus easily deal with complex geometries and heterogeneous propagation media. They also define the natural route to the so-called $h p$-adaptive solution strategies $\left[\mathrm{DKP}^{+} 07\right]$. Unfortunately, local mesh refinement can translate in a very restrictive time step in order to preserve the stability of the explicit time integration schemes which are most often adopted in FETD and DGTD methods. There are basically two directions to cure this efficiency problem. The first one consists in using a local time stepping strategy combined to an explicit time integration scheme, while the second approach relies on the use of an implicit or a hybrid explicit-implicit time integration scheme.

Local time stepping schemes have been studied by several authors. Fumeaux et al. [FBLV04] have designed such a strategy for a dissipative FVTD method on a non-uniform tetrahedral mesh. Their local time stepping scheme assumes a partitioning of the underlying mesh based on local geometrical properties while ensuring local stability. In each subdomain, the applied time step is some power of two of the smallest time step. In [FBLV04], the proposed local time stepping strategy is applied in conjunction with a Lax-Wendroff time advancing scheme but, according to the authors, the developed ideas could be adapted to other time schemes as well. An alternative multi-class local time stepping scheme is proposed by Piperno in [Pip06] in the context of a non-dissipative Leap-Frog based DGTD method. Given that the Leap-Frog scheme can be reformulated as a three-step Verlet scheme, the proposed strategy is based on a recursive application of the Verlet scheme on a multi-class arrangement of the mesh cells where a time step $\Delta t / 2^{N-k}$ is used in class $k, N$ being the total number of classes. The two-class strategy of this family is proved to conserve a discrete electromagnetic energy and to be stable provided $\Delta t$ is small enough, while the local time stepping multi-class strategy is assessed numerically in the context of the solution of the 2D Maxwell's equations by a DGTD method on triangular meshes. A two-class strategy is also considered by Cohen al. [CFP06] in the framework of a non-dissipative DGTD method on hexahedral meshes. It makes use of interpolations to approximate unknown fields when updating the cells values within a given class, however interpolations are recognized to be too expensive in terms of computtaional time further motivating the development of multiclass local time stepping strategies. Such a scheme is then developed by essentially the same authors in [MPFC08] but this time in the context of a dissipative version of the DGTD method considered in [CFP06]. Moreover, this new strategy is constructed as an adaptation of the one proposed by Piperno in [Pip06] and in particular, it involves a recursive application of the Leap-Frog scheme on a multi-class arrangement of the mesh cells where a time step 
$\Delta t / 3^{N-k}$ is used in class $k$. The resulting multi-class local time stepping scheme requires $33 \%$ less computation than the Verlet-based recursive method. A remark that applies to all the multi-class local time stepping strategies discussed so far is that a stability criterion is difficult to obtain and in some cases a reduction on the smallest time step is necessary for long time stability. More recently, a promising local time stepping strategy of arbitrary high order has been proposed by Diaz and Grote [DG07] for a second order scalar wave equation discretized in space by either a continuous or a discontinuous finite element method. The proposed strategy is based on the second order Leap-Frog scheme which is extended to arbitrarily high order by a modified equation approach. The resulting local time stepping method is proved to conserve a discrete energy and explicit CFL conditions are exhibited for the second order and fourth order accurate in time methods. According to the authors, this strategy extends easily to the Maxwell equations in second order form and for various discretization in space methods.

A few implicit variants of Yee's FDTD method have been developed among which, the alternating direction implicit finite difference time domain (ADI-FDTD) method [Nam00] which is a non-dissipative implicit FDTD method. The ADI-FDTD method offers unconditional stability with modest computational overhead despite its implicit formulation because it relies on a factorization of the implicit matrix operator leading to the inversion of tri-diagonal linear systems. A comprehensive analysis of the numerical dispersion of the ADI-FDTD method is presented in [ZC01] where it is shown that, on a non-uniform cartesian mesh, the time step can be taken uniformly the same as that defined by the coarsest cell without altering the accuracy in the finer mesh regions. However, in [GLH02], Garcia at al. exhibit accuracy limitations of the ADI-FDTD method that have not been revealed by previous studies on numerical dispersion, by investigating the truncation error on the time step due to the factorization. More precisely, the ADI-FDTD method is expressed as a $O\left(\Delta t^{2}\right)$ perturbation of an implicit Crank-Nicolson FDTD formulation and it is shown that some terms of the truncation error grow with the square of the time increment multiplied by the spatial derivatives of the fields, thus giving rise to potentially large numerical errors as the time step is increased. The development of implicit discontinuous Galerkin methods for the solution of time dependent problems has been less impressive than for their explicit counterparts. In [CDL09], an implicit DGTD method is proposed for the solution of the solution of the 2D Maxwell equations on triangular meshes. This method combines an arbitrary high order discontinuous Galerkin method for the discretization in space with a second order Cranck-Nicolson scheme for time integration. At each time step, a multifrontal sparse LU method is used for solving the linear system resulting from the discretization in space. When the simulations involve locally refined meshes and despite the computational overhead of the solution of a linear system at each time step, the resulting implicit DGTD method allows for a noticeable reduction of the computing time with regards to its explicit counterpart based on a Leap-Frog scheme, for comparable accuracy levels. However, in the $3 \mathrm{D}$ case, a globally implicit method based on a sparse direct solver suffers from large memory overheads. 
Explicit-implicit methods for the solution of the system of Maxwell equations have been studied by several authors with the shared goal of designing numerical methodologies able to deal with hybrid structured-unstructured meshes. For example, a stable hybrid FDTDFETD method is considered by Rylander and Bondeson in [RB02], while Degerfeldt and Rylander [DR06] propose a FETD method with stable hybrid explicit-implicit time stepping working on brick-tetrahedral meshes that do not require an intermediate layer of pyramidal elements. The implicit Newmark time stepping scheme is employed for the tetrahedral elements which allows for local mesh refinement while avoiding a reduced time step. For the brick elements, spatial lumping and explicit time stepping is employed, resulting in the application of the standard finite-difference time domain scheme. In [KCGH07], the authors study the application of explicit-implicit Runge-Kutta (so-called IMEX-RK) methods in conjunction with high order discontinuous Galerkin discretizations on unstructured triangular meshes, in the framework of unsteady compressible flow problems (i.e. the numerical solution of Euler or Navier-Stokes equations). Originally developed to solve the stiff operator of convection-diffusion-reaction models, IMEX-RK methods are in this work used for separating the time integration of stiff and non-stiff portions of the computational domain with regards to grid-induced stiffness. Another notable feature of this work is the application of automatic error-based time step controllers exploiting the fact that IMEX-RK methods provide embedded schemes which allow for the evaluation of a temporal error.

The present work is concerned with the development of a non-dissipative hybrid explicitimplicit DGTD method for solving the time domain Maxwell equations on unstructured simplicial meshes. The hybrid explicit-implicit DGTD method considered here has been initially introduced by Piperno [Pip06]. However, to our knowledge, this hybrid explicitimplicit DGTD method has not been investigated numerically so far for the simulation of realistic electromagnetic wave propagation problems. We conduct such a numerical investigation here for 2D and 3D propagation problems in homogeneous and heterogeneous media. We also propose a complete stability analysis of the method based on energetic considerations, extending a partial result obtained in [Pip06]. The rest of the paper is organized as follows: in section 2, we state the initial and boundary value problem to be solved; the discretization in space by a discontinous Galerkin method is discussed in section 3 while the integration in time is considered in section 4 ; the stability of the hybrid explicit-implicit DGTD method is treated in section 5; numerical results for $2 \mathrm{D}$ and $3 \mathrm{D}$ problems are respectively reported in sections 6 and 7 ; finally, section 8 concludes this paper and discusses future works.

\section{Continuous problem}

We consider the Maxwell equations in three space dimensions for heterogeneous linear isotropic media with no source. The electric field $\vec{E}(\vec{x}, t)={ }^{t}\left(E_{x}, E_{y}, E_{z}\right)$ and the magnetic field $\vec{H}(\vec{x}, t)={ }^{t}\left(H_{x}, H_{y}, H_{z}\right)$ verify: 


$$
\left\{\begin{array}{l}
\epsilon \partial_{t} \vec{E}-\operatorname{curl} \vec{H}=-\vec{J} \\
\mu \partial_{t} \vec{H}+\operatorname{curl} \vec{E}=0
\end{array}\right.
$$

where the symbol $\partial_{t}$ denotes a time derivative and $\vec{J}(\vec{x}, t)$ is a current source term. These equations are set on a bounded polyhedral domain $\Omega$ of $\mathbb{R}^{3}$. The permittivity $\epsilon(\vec{x})$ and the magnetic permeability tensor $\mu(\vec{x})$ are varying in space, time-invariant and both positive functions. Our goal is to solve system (1) in a domain $\Omega$ with boundary $\partial \Omega=\Gamma_{a} \cup \Gamma_{m}$, where we impose the following boundary conditions:

$$
\left\{\begin{array}{l}
\vec{n} \times \vec{E}=0 \text { on } \Gamma_{m}, \\
\vec{n} \times \vec{E}-\sqrt{\frac{\mu}{\varepsilon}} \vec{n} \times(\vec{H} \times \vec{n})=\vec{n} \times \vec{E}_{\mathrm{inc}}-\sqrt{\frac{\mu}{\varepsilon}} \vec{n} \times\left(\vec{H}_{\mathrm{inc}} \times \vec{n}\right) \text { on } \Gamma_{a} .
\end{array}\right.
$$

Here $\vec{n}$ denotes the unit outward normal to $\partial \Omega$ and $\left(\vec{E}_{\text {inc }}, \vec{H}_{\text {inc }}\right)$ is a given incident field. The first boundary condition is called metallic (referring to a perfectly conducting surface) while the second condition is called absorbing and takes here the form of the SilverMüller condition which is a first order approximation of the exact absorbing boundary condition. This absorbing condition is applied on $\Gamma_{a}$ which represents an artificial truncation of the computational domain. Finally, system (1) is supplemented with initial conditions: $\vec{E}_{0}(\vec{x})=\vec{E}(\vec{x}, t)$ and $\vec{H}_{0}(\vec{x})=\vec{H}(\vec{x}, t)$.

\section{Discretization in space}

We consider a partition $\mathcal{T}_{h}$ of $\Omega$ into a set of tetrahedra $\tau_{i}$ of size $h_{i}$ with boundary $\partial \tau_{i}$ such that $h=\max _{\tau_{i} \in \mathcal{T}_{h}} h_{i}$. For each $\tau_{i}, V_{i}$ denotes its volume, and $\epsilon_{i}$ and $\mu_{i}$ are respectively the local electric permittivity and magnetic permeability of the medium, which are assumed constant inside the element $\tau_{i}$. For two distinct tetrahedra $\tau_{i}$ and $\tau_{k}$ in $\mathcal{T}_{h}$, the intersection $\tau_{i} \cap \tau_{k}$ is a triangle $a_{i k}$ which we will call interface. For each internal interface $a_{i k}$, we denote by $\mathrm{S}_{i k}$ the measure of $a_{i k}$ and by $\vec{n}_{i k}$ the unitary normal vector, oriented from $\tau_{i}$ to $\tau_{k}$. For the boundary interfaces, the index $k$ corresponds to a fictitious element outside the domain. We denote by $\mathcal{F}_{h}^{I}$ the union of all interior interfaces of $\mathcal{T}_{h}$, by $\mathcal{F}_{h}^{B}$ the union of all boundary interfaces of $\mathcal{T}_{h}$, and by $\mathcal{F}_{h}=\mathcal{F}_{h}^{I} \cup \mathcal{F}_{h}^{B}$. Furthermore, we identify $\mathcal{F}_{h}^{B}$ to $\partial \Omega$ since $\Omega$ is a polyhedron. Finally, we denote by $\mathcal{V}_{i}$ the set of indices of the elements which are neighbors of $\tau_{i}$ (having an interface in common). We also define the perimeter $P_{i}$ of $\tau_{i}$ by $P_{i}=\sum_{k \in \mathcal{V}_{i}} \mathrm{~S}_{i k}$.

We have the following geometrical property for all elements: $\sum_{k \in \mathcal{V}_{i}} \mathrm{~S}_{i k} \vec{n}_{i k}=0$.

In the following, to simplify the presentation, we wet $\vec{J}=0$. For a given partition $\mathcal{T}_{h}$, we seek approximate solutions to (1) in the finite dimensional subspace: 


$$
V_{p}\left(\mathcal{T}_{h}\right)=\left\{\vec{v} \in L^{2}(\Omega)^{3}: v_{k \mid \tau_{i}} \in \mathbb{P}_{p}\left(\tau_{i}\right), \text { for } k=1,3 \text { and } \forall \tau_{i} \in \mathcal{T}_{h}\right\},
$$

where $\mathbb{P}_{p}\left(\tau_{i}\right)$ denotes the space of nodal polynomial functions of degree at most $p$ inside the element $\tau_{i}$.

Following the discontinuous Galerkin approach, the electric and magnetic fields inside each finite element are searched for as linear combinations $\left(\overrightarrow{\mathbf{E}}_{i}, \overrightarrow{\mathbf{H}}_{i}\right)$ of linearly independent basis vector fields $\vec{\varphi}_{i j}, 1 \leq j \leq d$, where $d$ denotes the local number of degrees of freedom inside $\tau_{i}$. Let $\mathcal{P}=\operatorname{Span}\left(\vec{\varphi}_{i j}, 1 \leq j \leq d\right)$. The approximate fields $\left(\overrightarrow{\mathbf{E}}_{h}, \overrightarrow{\mathbf{H}}_{h}\right)$, defined by $\left(\forall i, \overrightarrow{\mathbf{E}}_{h \mid \tau_{i}}=\overrightarrow{\mathbf{E}}_{i}, \overrightarrow{\mathbf{H}}_{h \mid \tau_{i}}=\overrightarrow{\mathbf{H}}_{i}\right)$ are allowed to be completely discontinuous across element boundaries. For such a discontinuous field $\overrightarrow{\mathbf{U}}_{h}$, we define its average $\left\{\overrightarrow{\mathbf{U}}_{h}\right\}_{i k}$ through any internal interface $a_{i k}$, as $\left\{\overrightarrow{\mathbf{U}}_{h}\right\}_{i k}=\left(\overrightarrow{\mathbf{U}}_{i \mid a_{i k}}+\overrightarrow{\mathbf{U}}_{k \mid a_{i k}}\right) / 2$. Note that for any internal interface $a_{i k},\left\{\overrightarrow{\mathbf{U}}_{h}\right\}_{k i}=\left\{\overrightarrow{\mathbf{U}}_{h}\right\}_{i k}$. Because of this discontinuity, a global variational formulation cannot be obtained. However, dot-multiplying (1) by any given vector function $\vec{\varphi} \in \mathcal{P}$, integrating over each single element $\tau_{i}$ and integrating by parts, yields:

$$
\left\{\begin{array}{l}
\int_{\tau_{i}} \vec{\varphi} \cdot \epsilon_{i} \partial_{t} \overrightarrow{\mathbf{E}}=\int_{\tau_{i}} \operatorname{curl} \vec{\varphi} \cdot \overrightarrow{\mathbf{H}}-\int_{\partial \tau_{i}} \vec{\varphi} \cdot(\overrightarrow{\mathbf{H}} \times \vec{n}), \\
\int_{\tau_{i}} \vec{\varphi} \cdot \mu_{i} \partial_{t} \overrightarrow{\mathbf{H}}=-\int_{\tau_{i}} \operatorname{curl} \vec{\varphi} \cdot \overrightarrow{\mathbf{E}}+\int_{\partial \tau_{i}} \vec{\varphi} \cdot(\overrightarrow{\mathbf{E}} \times \vec{n}) .
\end{array}\right.
$$

In Eq. (4), we now replace the exact fields $\overrightarrow{\mathbf{E}}$ and $\overrightarrow{\mathbf{H}}$ by the approximate fields $\overrightarrow{\mathbf{E}}_{h}$ and $\overrightarrow{\mathbf{H}}_{h}$ in order to evaluate volume integrals. For integrals over $\partial \tau_{i}$, a specific treatment must be introduced since the approximate fields are discontinuous through element faces, leading to the definition of a numerical flux. We choose to use a fully centered numerical flux, i.e. $\forall i, \forall k \in \mathcal{V}_{i}, \overrightarrow{\mathbf{E}}_{\mid a_{i k}} \simeq\left\{\overrightarrow{\mathbf{E}}_{h}\right\}_{i k}, \overrightarrow{\mathbf{H}}_{\mid a_{i k}} \simeq\left\{\overrightarrow{\mathbf{H}}_{h}\right\}_{i k}$. The metallic boundary condition (first relation of (2)) on a boundary interface $a_{i k} \in \Gamma_{m}$ ( $k$ in the element index of the fictitious neighboring element) is dealt with weakly, in the sense that traces of fictitious fields $\overrightarrow{\mathbf{E}}_{k}$ and $\overrightarrow{\mathbf{H}}_{k}$ are used for the computation of numerical fluxes for the boundary element $\tau_{i}$. More precisely, we set $\overrightarrow{\mathbf{E}}_{k \mid a_{i k}}=-\overrightarrow{\mathbf{E}}_{i \mid a_{i k}}$ and $\overrightarrow{\mathbf{H}}_{k \mid a_{i k}}=\overrightarrow{\mathbf{H}}_{i \mid a_{i k}}$. Similarly, the absorbing boundary condition (second relation of (2)) is taken into account through the use of a fully upwind numerical flux for the evaluation of the corresponding boundary integral over $a_{i k} \in \Gamma_{a}$ (see [CDL09] for more details). From now on, we assume $\Gamma_{a}=\emptyset$ except in the numerical results section where we consider both internal (i.e. cavity) and external propagation problems. Evaluating the surface integrals in (4) using the centered numerical flux, and re-integrating by parts yields: 


$$
\left\{\begin{aligned}
\int_{\tau_{i}} \vec{\varphi} \cdot \epsilon_{i} \partial_{t} \overrightarrow{\mathbf{E}}_{i} & =\frac{1}{2} \int_{\tau_{i}}\left(\operatorname{curl} \vec{\varphi} \cdot \overrightarrow{\mathbf{H}}_{i}+\operatorname{curl} \overrightarrow{\mathbf{H}}_{i} \cdot \vec{\varphi}\right) \\
& -\frac{1}{2} \sum_{k \in \mathcal{V}_{i}} \int_{a_{i k}} \vec{\varphi} \cdot\left(\overrightarrow{\mathbf{H}}_{k} \times \vec{n}_{i k}\right), \\
\int_{\tau_{i}} \vec{\varphi} \cdot \mu_{i} \partial_{t} \overrightarrow{\mathbf{H}}_{i} & =-\frac{1}{2} \int_{\tau_{i}}\left(\operatorname{curl} \vec{\varphi} \cdot \overrightarrow{\mathbf{E}}_{i}+\operatorname{curl} \overrightarrow{\mathbf{E}}_{i} \cdot \vec{\varphi}\right) \\
& +\frac{1}{2} \sum_{k \in \mathcal{V}_{i}} \int_{a_{i k}} \vec{\varphi} \cdot\left(\overrightarrow{\mathbf{E}}_{k} \times \vec{n}_{i k}\right) .
\end{aligned}\right.
$$

Eq. (5) can be rewritten in terms of scalar unknowns. Inside each element, the fields are re-composed according to $\overrightarrow{\mathbf{E}}_{i}=\sum_{1 \leq j \leq d} E_{i j} \vec{\varphi}_{i j}$ and $\overrightarrow{\mathbf{H}}_{i}=\sum_{1 \leq j \leq d} H_{i j} \vec{\varphi}_{i j}$ and let us now denote by $\mathbf{E}_{i}$ and $\mathbf{H}_{i}$ respectively the column vectors $\left(E_{i l}\right)_{1 \leq l \leq d}$ and $\left(H_{i l}\right)_{1 \leq l \leq d}$. Then, (5) is equivalent to:

$$
\left\{\begin{aligned}
M_{i}^{\epsilon} \frac{d \mathbf{E}_{i}}{d t} & =K_{i} \mathbf{H}_{i}-\sum_{k \in \mathcal{V}_{i}} S_{i k} \mathbf{H}_{k} \\
M_{i}^{\mu} \frac{d \mathbf{H}_{i}}{d t} & =-K_{i} \mathbf{E}_{i}+\sum_{k \in \mathcal{V}_{i}} S_{i k} \mathbf{E}_{k}
\end{aligned}\right.
$$

where the symmetric positive definite mass matrices $M_{i}^{\sigma}$ ( $\sigma$ stands for $\epsilon$ or $\mu$ ), the symmetric stiffness matrix $K_{i}$ and the symmetric interface matrix $S_{i k}$ (all of size $d \times d$ ) are given by:

$$
\begin{aligned}
\left(M_{i}^{\sigma}\right)_{j l} & =\sigma_{i} \int_{\tau_{i}}{ }^{t} \vec{\varphi}_{i j} \cdot \vec{\varphi}_{i l}, \\
\left(K_{i}\right)_{j l} & =\frac{1}{2} \int_{\tau_{i}}{ }^{t} \vec{\varphi}_{i j} \cdot \operatorname{curl} \vec{\varphi}_{i l}+{ }^{t} \vec{\varphi}_{i l} \cdot \operatorname{curl} \vec{\varphi}_{i j}, \\
\left(S_{i k}\right)_{j l} & =\frac{1}{2} \int_{a_{i k}}{ }^{t} \vec{\varphi}_{i j} \cdot\left(\vec{\varphi}_{k l} \times \vec{n}_{i k}\right) .
\end{aligned}
$$

\section{Time discretization}

The choice of the time discretization method is a crucial step for the global efficiency of the numerical method. The temporal integration methods are divided into two major families: implicit and explicit schemes. Implicit schemes require the solution of large matrix systems resulting in a high computational effort per time iteration and the viability of such a scheme strongly depends on the efficiency of the used linear system solver. The advantage of implicit schemes is their robustness concerning the choice of the time step used than can be chosen

RR $n^{\circ} 6990$ 
arbitrarily large in the case of an unconditionally stable scheme. Thus, a simulation requires only a small number of time iterations, but every time step is burdened by a high numerical effort. Explicit schemes in contrast are easy to implement, produce greater accuracy with less computational effort than implicit methods, but are restricted by a stability criterion enforcing a close linkage of the time step to the spatial discretization parameter. This restriction may result in a large number of iterations per analysis, each iteration with a low computational effort. Then, a possible alternative is to combine the strengths of both schemes by applying an implicit time integration scheme locally in the refined regions of the mesh while preserving an explicit time scheme in the complementary part, resulting in an hybrid explicit-implicit (or locally implicit) time integration strategy.

The set of local system of ordinary differential equations for each $\tau_{i}(6)$ can be formally transformed in a global system. To this end, we suppose that all electric (resp. magnetic) unknowns are gathered in a column vector $\mathbb{E}$ (resp. $\mathbb{H}$ ) of size $d_{g}=N_{\mathcal{T}_{h}} d$ where $N_{\mathcal{T}_{h}}$ stands for the number of elements in $\mathcal{T}_{h}$. Then system (6) can be rewritten as:

$$
\left\{\begin{array}{l}
\mathbb{M}^{\frac{\epsilon}{\epsilon}} \frac{d \mathbb{E}}{d t}=\mathbb{K} \mathbb{H}-\mathbb{A} \mathbb{H}-\mathbb{B H}, \\
\mathbb{M}^{\mu} \frac{d \mathbb{H}}{d t}=-\mathbb{K} \mathbb{E}+\mathbb{A} \mathbb{E}-\mathbb{B E},
\end{array}\right.
$$

where we have the following definitions and properties:

- $\mathbb{M}^{\epsilon}, \mathbb{M}^{\mu}$ and $\mathbb{K}$ are $d_{g} \times d_{g}$ block diagonal matrices with diagonal blocks equal to $M_{i}^{\epsilon}, M_{i}^{\mu}$ and $K_{i}$ respectively. Therefore $\mathbb{M}^{\epsilon}$ and $\mathbb{M}^{\mu}$ are symmetric positive definite matrices, and $\mathbb{K}$ is a symmetric matrix.

- $\mathbb{A}$ is also a $d_{g} \times d_{g}$ block sparse matrix, whose non-zero blocks are equal to $S_{i k}$ when $a_{i k} \in \mathcal{F}_{h}^{I}$. Since $\vec{n}_{k i}=-\vec{n}_{i k}$, it can be checked that $\left(S_{i k}\right)_{j l}=\left(S_{k i}\right)_{l j}$ and then $S_{k i}={ }^{t} S_{i k}$; thus $\mathbb{A}$ is a symmetric matrix.

- $\mathbb{B}$ is a $d_{g} \times d_{g}$ block diagonal matrix, whose non-zero blocks are equal to $S_{i k}$ when $a_{i k} \in \mathcal{F}_{h}^{B}$. In that case, $\left(S_{i k}\right)_{j l}=-\left(S_{i k}\right)_{l j}$; thus $\mathbb{B}$ is a skew-symmetric matrix.

Consequently, if we set $\mathbb{S}=\mathbb{K}-\mathbb{A}-\mathbb{B}$, the system (7) rewrites as:

$$
\left\{\begin{array}{l}
\mathbb{M}^{\epsilon} \frac{d \mathbb{E}}{d t}=\mathbb{S H} \\
\mathbb{M}^{\mu} \frac{d \mathbb{H}}{d t}=-{ }^{t} \mathbb{S E}
\end{array}\right.
$$

\subsection{Explicit time scheme}

The semi-discrete system (8) can be time integrated using a second-order Leap-Frog scheme as: 


$$
\left\{\begin{array}{l}
\mathbb{M}^{\epsilon}\left(\frac{\mathbb{E}^{n+1}-\mathbb{E}^{n}}{\Delta t}\right)=\mathbb{S H}^{n+\frac{1}{2}} \\
\mathbb{M}^{\mu}\left(\frac{\mathbb{H}^{n+\frac{3}{2}}-\mathbb{H}^{n+\frac{1}{2}}}{\Delta t}\right)=-{ }^{t} \mathbb{S E}^{n+1} .
\end{array}\right.
$$

The resulting fully explicit DGTD- $\mathbb{P}_{p}$ method is analyzed in [FLLP05] where it is shown that the method is non-dissipative, conserves a discrete form of the electromagnetic energy and is stable under the CFL-like condition:

$$
\Delta t \leq \frac{2}{\alpha}, \quad \text { with } \alpha=\left\|\left(\mathbb{M}^{-\mu}\right)^{\frac{1}{2}}{ }^{t} \mathbb{S}\left(\mathbb{M}^{-\epsilon}\right)^{\frac{1}{2}}\right\|,
$$

where the matrix $\left(\mathbb{M}^{\sigma}\right)^{-\frac{1}{2}}$ is the inverse square root of $\mathbb{M}^{\sigma}$.

\subsection{Implicit time scheme}

Alternatively, the semi-discrete system (8) can be time integrated using a second-order Crank-Nicolson scheme as:

$$
\left\{\begin{array}{l}
\mathbb{M}^{\epsilon}\left(\frac{\mathbb{E}^{n+1}-\mathbb{E}^{n}}{\Delta t}\right)=\mathbb{S}\left(\frac{\mathbb{H}^{n}+\mathbb{H}^{n+1}}{2}\right), \\
\mathbb{M}^{\mu}\left(\frac{\mathbb{H}^{n+1}-\mathbb{H}^{n}}{\Delta t}\right)=-{ }^{t} \mathbb{S}\left(\frac{\mathbb{E}^{n}+\mathbb{E}^{n+1}}{2}\right) .
\end{array}\right.
$$

Such a fully implicit DGTD- $\mathbb{P}_{p}$ method is considered in [CDL09] for the solution of the 2D Maxwell equations. In particular, the resulting method is unconditionally stable.

\subsection{Hybrid explicit-implicit time scheme}

As mentioned above, explicit and implicit time scheme based methods have their own advantages and drawbacks. When the underlying mesh is locally refined a more viable approach consists in applying an implicit time scheme locally in the refined regions of the mesh, while preserving an explicit time scheme in the complementary part, resulting in an hybrid explicit-implicit (or locally implicit) time integration strategy. We consider here a method of this kind that was recently proposed by Piperno in [Pip06]. The set of elements $\tau_{i}$ of the mesh is now assumed to be partitioned into two subsets: one made of the smallest elements and the other one gathering the remaining elements. In the following, these two subsets are respectively referred as $\mathcal{S}_{i}$ and $\mathcal{S}_{e}$. The distinction between the two subsets can be done according to a geometrical threshold, or/and a physical criterion as well. Note that there is no need of a particular assumption on the connectivity of the two subsets. In the proposed hybrid time scheme, the small elements are handled using a Crank-Nicolson scheme while all other elements are time advanced using a variant of the classical Leap-Frog scheme known as the Verlet method. In the Verlet method, the fields $\mathbb{H}$ and $\mathbb{E}$ are defined at the same time 
station and time integration proceeds in three sub-steps: (1) $\mathbb{H}$ is time advanced from $t^{n}$ to $t^{n+\frac{1}{2}}$ with time step $\Delta t / 2,(2) \mathbb{E}$ is time advanced from $t^{n}$ to $t^{n+1}$ with time step $\Delta t$ and, (3) $\mathbb{H}$ is time advanced from $t^{n+\frac{1}{2}}$ to $t^{n+1}$ with time step $\Delta t / 2$. Then, starting from the values of the fields at time $t^{n}=n \Delta t$, the proposed hybrid explicit-implicit time integration scheme consists in three sub-steps:

(1) the components of $\mathbb{H}$ and $\mathbb{E}$ associated to the set $\mathcal{S}_{e}$ are time advanced from $t^{n}$ to $t^{n+\frac{1}{2}}$ with time step $\Delta t / 2$ using a pseudo-forward Euler scheme,

(2) the components of $\mathbb{H}$ and $\mathbb{E}$ associated to the set $\mathcal{S}_{i}$ are time advanced from $t^{n}$ to $t^{n+1}$ with time step $\Delta t$ using the Crank-Nicolson scheme,

(1) the components of $\mathbb{H}$ and $\mathbb{E} 1$ associated to the set $\mathcal{S}_{e}$ are time advanced from $t^{n+\frac{1}{2}}$ to $t^{n+1}$ with time step $\Delta t / 2$ using the reversed pseudo-forward Euler scheme.

In order to further describe this scheme, we introduce additional definitions. First, the problem unknowns are reordered as:

$$
\mathbb{E}=\left(\begin{array}{c}
\mathbb{E}_{e} \\
\mathbb{E}_{i}
\end{array}\right) \quad \text { and } \quad \mathbb{H}=\left(\begin{array}{c}
\mathbb{H}_{e} \\
\mathbb{H}_{i}
\end{array}\right)
$$

where sub-vectors with an $e$ subscript (respectively, an $i$ subscript) are associated to the clements of the set $\mathcal{S}_{e}$ (respectively, the set $\mathcal{S}_{i}$ ). We deduce from this partitioning of the unknown vectors the following decompositions of the system matrices:

$$
\begin{gathered}
\mathbb{M}^{\varepsilon}=\left(\begin{array}{cc}
\mathbb{M}_{e}^{\varepsilon} & \mathbb{O} \\
\mathbb{O} & \mathbb{M}_{\varepsilon}^{i}
\end{array}\right), \mathbb{M}^{\mu}=\left(\begin{array}{cc}
\mathbb{M}_{e}^{\mu} & \mathbb{O} \\
\mathbb{O} & \mathbb{M}_{i}^{\mu}
\end{array}\right) \\
\mathbb{K}=\left(\begin{array}{cc}
\mathbb{K}_{e} & \mathbb{O} \\
\mathbb{O} & \mathbb{K}_{i}
\end{array}\right), \mathbb{B}=\left(\begin{array}{cc}
\mathbb{B}_{e} & \mathbb{O} \\
\mathbb{O} & \mathbb{B}_{i}
\end{array}\right) .
\end{gathered}
$$

where $\mathbb{M}_{e / i}^{\varepsilon}$ and $\mathbb{M}_{e / i}^{\mu}$ are symmetric positive definite matrices, $\mathbb{K}_{e / i}$ are symmetric matrices and $\mathbb{B}_{e / i}$ are skew-symmetric matrices. The matrix $\mathbb{A}$ which involves the interface matrices $S_{i k}$ is decomposed as:

$$
\mathbb{A}=\left(\begin{array}{cc}
\mathbb{A}_{e e} & \mathbb{A}_{e i} \\
\mathbb{A}_{i e} & \mathbb{A}_{i i}
\end{array}\right)
$$

where $\mathbb{A}_{e e}$ and $\mathbb{A}_{i i}$ are symmetric matrices, and $\mathbb{A}_{e i}={ }^{t} \mathbb{A}_{i e}$. Introducing the two matrices $\mathbb{S}_{e}=\mathbb{K}_{e}-\mathbb{A}_{e e}-\mathbb{B}_{e}$ and $\mathbb{S}_{i}=\mathbb{K}_{i}-\mathbb{A}_{i i}-\mathbb{B}_{i}$, the global system of ordinary differential equations (8) can be split into two systems: 


$$
\begin{aligned}
& \left\{\begin{array}{l}
\mathbb{M}_{e}^{\varepsilon} \frac{d \mathbb{E}_{e}}{d t}=\mathbb{S}_{e} \mathbb{H}_{e}-\mathbb{A}_{e i} \mathbb{H}_{i}, \\
\mathbb{M}_{e}^{\mu} \frac{d \mathbb{H}_{e}}{d t}=-{ }^{t} \mathbb{S}_{e} \mathbb{E}_{e}+\mathbb{A}_{e i} \mathbb{E}_{i},
\end{array}\right. \\
& \left\{\begin{array}{l}
\mathbb{M}_{i}^{\varepsilon} \frac{d \mathbb{E}_{i}}{d t}=\mathbb{S}_{i} \mathbb{H}_{i}-\mathbb{A}_{i e} \mathbb{H}_{e}, \\
\mathbb{M}_{i}^{\mu} \frac{d \mathbb{H}_{i}}{d t}=-{ }^{t} \mathbb{S}_{i} \mathbb{E}_{i}+\mathbb{A}_{i e} \mathbb{E}_{e}
\end{array}\right.
\end{aligned}
$$

Then, the proposed hybrid explicit-implicit algorithm consists in the following steps:

$$
\begin{aligned}
& \left\{\begin{array}{l}
\mathbb{M}_{e}^{\mu}\left(\frac{\mathbb{H}_{e}^{n+\frac{1}{2}}-\mathbb{H}_{e}^{n}}{\Delta t / 2}\right)=-{ }^{t} \mathbb{S}_{e} \mathbb{E}_{e}^{n}+\mathbb{A}_{e i} \mathbb{E}_{i}^{n}, \\
\mathbb{M}_{e}^{\varepsilon}\left(\frac{\mathbb{E}_{e}^{n+\frac{1}{2}}-\mathbb{E}_{e}^{n}}{\Delta t / 2}\right)=\mathbb{S}_{e} \mathbb{H}_{e}^{n+\frac{1}{2}}-\mathbb{A}_{e i} \mathbb{H}_{i}^{n},
\end{array}\right. \\
& \left\{\begin{array}{l}
\mathbb{M}_{i}^{\varepsilon}\left(\frac{\mathbb{E}_{i}^{n+1}-\mathbb{E}_{i}^{n}}{\Delta t}\right)=\mathbb{S}_{i}\left(\frac{\mathbb{H}_{i}^{n+1}+\mathbb{H}_{i}^{n}}{2}\right)-\mathbb{A}_{i e} \mathbb{H}_{e}^{n+\frac{1}{2}}, \\
\mathbb{M}_{i}^{\mu}\left(\frac{\mathbb{H}_{i}^{n+1}-\mathbb{H}_{i}^{n}}{\Delta t}\right)=-{ }^{t} \mathbb{S}_{i}\left(\frac{\mathbb{E}_{i}^{n+1}+\mathbb{E}_{i}^{n}}{2}\right)+\mathbb{A}_{i e} \mathbb{E}_{e}^{n+\frac{1}{2}}
\end{array}\right. \\
& \left\{\begin{array}{l}
\mathbb{M}_{e}^{\varepsilon}\left(\frac{\mathbb{E}_{e}^{n+1}-\mathbb{E}_{e}^{n+\frac{1}{2}}}{\Delta t / 2}\right)=\mathbb{S}_{e} \mathbb{H}_{e}^{n+\frac{1}{2}}-\mathbb{A}_{e i} \mathbb{H}_{i}^{n+1}, \\
\mathbb{M}_{e}^{\mu}\left(\frac{\mathbb{H}_{e}^{n+1}-\mathbb{H}_{e}^{n+\frac{1}{2}}}{\Delta t / 2}\right)=-{ }^{t} \mathbb{S}_{e} \mathbb{E}_{e}^{n+1}+\mathbb{A}_{e i} \mathbb{E}_{i}^{n+1} .
\end{array}\right.
\end{aligned}
$$

\section{Stability study of the hybrid explicit-implicit DGTD- $\mathbb{P}_{p}$ method}

In [Pip06], the author shows that the hybrid explicit-implicit scheme (13) for time integration of the semi-discrete system (8) associated to the DGTD- $\mathbb{P}_{p}$ method exactly conserves the following quadratic form of the numerical unknowns $\mathbb{E}_{e}^{n}, \mathbb{E}_{i}^{n}, \mathbb{H}_{e}^{n}$ and $\mathbb{H}_{i}^{n}$ : 


$$
\mathcal{E}^{n}=\mathcal{E}_{e}^{n}+\mathcal{E}_{i}^{n}+\mathcal{E}_{h}^{n} \quad \text { with }\left\{\begin{aligned}
\mathcal{E}_{e}^{n} & ={ }^{t} \mathbb{E}_{e}^{n} \mathbb{M}_{e}^{\varepsilon} \mathbb{E}_{e}^{n}+{ }^{t} \mathbb{H}_{e}^{n+\frac{1}{2}} \mathbb{M}_{e}^{\mu} \mathbb{H}_{e}^{n-\frac{1}{2}}, \\
\mathcal{E}_{i}^{n} & ={ }^{t} \mathbb{E}_{i}^{n} \mathbb{M}_{i}^{\varepsilon} \mathbb{E}_{i}^{n}+{ }^{t} \mathbb{H}_{i}^{n} \mathbb{M}_{i}^{\mu} \mathbb{H}_{i}^{n}, \\
\mathcal{E}_{h}^{n} & =-\frac{\Delta t^{2}}{4}{ }^{t} \mathbb{H}_{i}^{n}{ }^{t} \mathbb{A}_{e i}\left(\mathbb{M}_{e}^{\varepsilon}\right)^{-1} \mathbb{A}_{e i} \mathbb{H}_{i}^{n},
\end{aligned}\right.
$$

as far as $\Gamma_{a}=\emptyset$. However, the condition under which $\mathcal{E}^{n}$ is a positive definite quadratic form and thus represents a discrete form of the electromagnetic energy is not given. In the following we state a condition on the global time step $\Delta t$ such that $\mathcal{E}^{n}$ is a positive definite quadratic form.

Lemma 1 The discrete electromagnetic energy $\mathcal{E}^{n}$ given by Eq. (14) is a positive definite quadratic form of the numerical unknowns $\mathbb{E}_{e}^{n}, \mathbb{E}_{i}^{n}, \mathbb{H}_{e}^{n}$ and $\mathbb{H}_{i}^{n}$ if:

$$
\Delta t \leq \frac{2}{\alpha_{e}+\max \left(\beta_{e i}, \gamma_{e i}\right)} \quad \text { with }\left\{\begin{array}{l}
\alpha_{e}=\left\|\left(\mathbb{M}_{e}^{\varepsilon}\right)^{-\frac{1}{2}} \mathbb{S}_{e}\left(\mathbb{M}_{e}^{\mu}\right)^{-\frac{1}{2}}\right\|, \\
\beta_{e i}=\left\|\left(\mathbb{M}_{e}^{\varepsilon}\right)^{-\frac{1}{2}} \mathbb{A}_{e i}\left(\mathbb{M}_{i}^{\mu}\right)^{-\frac{1}{2}}\right\|, \\
\gamma_{e i}=\left\|\left(\mathbb{M}_{e}^{\mu}\right)^{-\frac{1}{2}} \mathbb{A}_{e i}\left(\mathbb{M}_{i}^{\varepsilon}\right)^{-\frac{1}{2}}\right\|,
\end{array}\right.
$$

where $\|\cdot\|$ denotes a matrix norm and the matrix $\left(\mathbb{M}_{e / i}^{\sigma}\right)^{-\frac{1}{2}}$ is the inverse of the square root of the matrix $\mathbb{M}_{e / i}^{\sigma}$ ( $\sigma$ stands for $\varepsilon$ or $\mu$ ).

Proof. We rewrite the last and the first relations of the hybrid explicit-implicit algorithm (13) respectively as:

$$
\left\{\begin{array}{l}
\mathbb{M}_{e}^{\mu} \mathbb{H}_{e}^{n-\frac{1}{2}}=\mathbb{M}_{e}^{\mu} \mathbb{H}_{e}^{n}-\frac{\Delta t}{2}\left(-{ }^{t} \mathbb{S}_{e} \mathbb{E}_{e}^{n}+\mathbb{A}_{e i} \mathbb{E}_{i}^{n}\right), \\
\mathbb{M}_{e}^{\mu} \mathbb{H}_{e}^{n+\frac{1}{2}}=\mathbb{M}_{e}^{\mu} \mathbb{H}_{e}^{n}+\frac{\Delta t}{2}\left(-{ }^{t} \mathbb{S}_{e} \mathbb{E}_{e}^{n}+\mathbb{A}_{e i} \mathbb{E}_{i}^{n}\right) .
\end{array}\right.
$$

Multiplying the first relation of (16) by ${ }^{t} \mathbb{H}_{e}^{n+\frac{1}{2}}$ and using the expression of ${ }^{t} \mathbb{H}_{e}^{n+\frac{1}{2}}$ from the second relation of (16) leads to:

$$
\begin{aligned}
{ }^{t} \mathbb{H}_{e}^{n+\frac{1}{2}} \mathbb{M}_{e}^{\mu} \mathbb{H}_{e}^{n-\frac{1}{2}} & ={ }^{t} \mathbb{H}_{e}^{n} \mathbb{M}_{e}^{\mu} \mathbb{H}_{e}^{n} \\
& -\frac{\Delta t^{2}}{4}{ }^{t}\left(-{ }^{t} \mathbb{S}_{e} \mathbb{E}_{e}^{n}+\mathbb{A}_{e i} \mathbb{E}_{i}^{n}\right)\left(\mathbb{M}_{e}^{\mu}\right)^{-1}\left(-{ }^{t} \mathbb{S}_{e} \mathbb{E}_{e}^{n}+\mathbb{A}_{e i} \mathbb{E}_{i}^{n}\right)
\end{aligned}
$$

Then, the explicit part of the discrete electromagnetic energy (14) yields: 


$$
\begin{aligned}
\mathcal{E}_{e}^{n}=\left\|\left(\mathbb{M}_{e}^{\varepsilon}\right)^{\frac{1}{2}} \mathbb{E}_{e}^{n}\right\|^{2} & +{ }^{t} \mathbb{H}_{e}^{n+\frac{1}{2}} \mathbb{M}_{e}^{\mu} \mathbb{H}_{e}^{n-\frac{1}{2}} \\
=\left\|\left(\mathbb{M}_{e}^{\varepsilon}\right)^{\frac{1}{2}} \mathbb{E}_{e}^{n}\right\|^{2} & +\left\|\left(\mathbb{M}_{e}^{\mu}\right)^{\frac{1}{2}} \mathbb{H}_{e}^{n}\right\|^{2} \\
& \quad-\frac{\Delta t^{2}}{4}\left\|\left(\mathbb{M}_{e}^{\mu}\right)^{-\frac{1}{2}}\left(-{ }^{t} \mathbb{S}_{e} \mathbb{E}_{e}^{n}+\mathbb{A}_{e i} \mathbb{E}_{i}^{n}\right)\right\|^{2} .
\end{aligned}
$$

Denting by $\Pi=\left\|\left(\mathbb{M}_{e}^{\mu}\right)^{-\frac{1}{2}}\left(-{ }^{t} \mathbb{S}_{e} \mathbb{E}_{e}^{n}+\mathbb{A}_{e i} \mathbb{E}_{i}^{n}\right)\right\|^{2}$, we have that:

$$
\begin{aligned}
\Pi \leq & \left\|\left(\mathbb{M}_{e}^{\mu}\right)^{-\frac{1}{2} t} \mathbb{S}_{e} \mathbb{E}_{e}^{n}\right\|^{2}+\left\|\left(\mathbb{M}_{e}^{\mu}\right)^{-\frac{1}{2}} \mathbb{A}_{e i} \mathbb{E}_{i}^{n}\right\|^{2}+ \\
& 2\left\|\left(\mathbb{M}_{e}^{\mu}\right)^{-\frac{1}{2} t} \mathbb{S}_{e} \mathbb{E}_{e}^{n}\right\|\left\|\left(\mathbb{M}_{e}^{\mu}\right)^{-\frac{1}{2}} \mathbb{A}_{e i} \mathbb{E}_{i}^{n}\right\| \\
\leq & \alpha_{e}^{2}\left\|\left(\mathbb{M}_{e}^{\varepsilon}\right)^{\frac{1}{2}} \mathbb{E}_{e}^{n}\right\|^{2}+\gamma_{e i}^{2}\left\|\left(\mathbb{M}_{i}^{\varepsilon}\right)^{\frac{1}{2}} \mathbb{E}_{i}^{n}\right\|^{2}+ \\
& 2 \alpha_{e} \gamma_{e i}\left\|\left(\mathbb{M}_{e}^{\varepsilon}\right)^{\frac{1}{2}} \mathbb{E}_{e}^{n}\right\|\left\|\left(\mathbb{M}_{i}^{\varepsilon}\right)^{\frac{1}{2}} \mathbb{E}_{i}^{n}\right\| \\
\leq & \left(\alpha_{e}^{2}+\alpha_{e} \gamma_{e i}\right)\left\|\left(\mathbb{M}_{e}^{\varepsilon}\right)^{\frac{1}{2}} \mathbb{E}_{e}^{n}\right\|^{2}+\left(\gamma_{e i}^{2}+\alpha_{e} \gamma_{e i}\right)\left\|\left(\mathbb{M}_{i}^{\varepsilon}\right)^{\frac{1}{2}} \mathbb{E}_{i}^{n}\right\|^{2},
\end{aligned}
$$

where the last relation has been obtained using the inequality $2 a b \leq a^{2}+b^{2}$. We deduce from (17) that:

$$
\begin{aligned}
\mathcal{E}_{e}^{n} \geq & {\left[1-\frac{\Delta t^{2}}{4}\left(\alpha_{e}^{2}+\alpha_{e} \gamma_{e i}\right)\right]\left\|\left(\mathbb{M}_{e}^{\varepsilon}\right)^{\frac{1}{2}} \mathbb{E}_{e}^{n}\right\|^{2}+} \\
& \left\|\left(\mathbb{M}_{e}^{\mu}\right)^{\frac{1}{2}} \mathbb{H}_{e}^{n}\right\|^{2}-\frac{\Delta t^{2}}{4}\left(\gamma_{e i}^{2}+\alpha_{e} \gamma_{e i}\right)\left\|\left(\mathbb{M}_{i}^{\varepsilon}\right)^{\frac{1}{2}} \mathbb{E}_{i}^{n}\right\|^{2} .
\end{aligned}
$$

For the implicit part of the discrete electromagnetic energy (14) we have:

$$
\mathcal{E}_{i}^{n}=\left\|\left(\mathbb{M}_{i}^{\varepsilon}\right)^{\frac{1}{2}} \mathbb{E}_{i}^{n}\right\|^{2}+\left\|\left(\mathbb{M}_{i}^{\mu}\right)^{\frac{1}{2}} \mathbb{H}_{i}^{n}\right\|^{2} .
$$

Gathering (18) and (19) yields:

$$
\begin{aligned}
\mathcal{E}_{e}^{n}+\mathcal{E}_{i}^{n} \geq & {\left[1-\frac{\Delta t^{2}}{4}\left(\alpha_{e}^{2}+\alpha_{e} \gamma_{e i}\right)\right]\left\|\left(\mathbb{M}_{e}^{\varepsilon}\right)^{\frac{1}{2}} \mathbb{E}_{e}^{n}\right\|^{2}+} \\
& {\left[1-\frac{\Delta t^{2}}{4}\left(\gamma_{e i}^{2}+\alpha_{e} \gamma_{e i}\right)\right]\left\|\left(\mathbb{M}_{i}^{\varepsilon}\right)^{\frac{1}{2}} \mathbb{E}_{i}^{n}\right\|^{2}+} \\
& \left\|\left(\mathbb{M}_{e}^{\mu}\right)^{\frac{1}{2}} \mathbb{H}_{e}^{n}\right\|^{2}+\left\|\left(\mathbb{M}_{i}^{\mu}\right)^{\frac{1}{2}} \mathbb{H}_{i}^{n}\right\|^{2}
\end{aligned}
$$

Using the simple relations: 


$$
\left\{\begin{array}{l}
\alpha_{e}^{2}+\alpha_{e} \gamma_{e i}=\left(\alpha_{e}+\gamma_{e i}\right)^{2}-\alpha_{e} \gamma_{e i}-\gamma_{e i}^{2} \\
\gamma_{e i}^{2}+\alpha_{e} \gamma_{e i}=\left(\alpha_{e}+\gamma_{e i}\right)^{2}-\alpha_{e} \gamma_{e i}-\alpha_{e}^{2}
\end{array}\right.
$$

we modify (20) as:

$$
\begin{aligned}
\mathcal{E}_{e}^{n}+\mathcal{E}_{i}^{n} \geq & {\left[1-\frac{\Delta t^{2}}{4}\left(\alpha_{e}+\gamma_{e i}\right)^{2}\right]\left\|\left(\mathbb{M}_{e}^{\varepsilon}\right)^{\frac{1}{2}} \mathbb{E}_{e}^{n}\right\|^{2}+} \\
& {\left[1-\frac{\Delta t^{2}}{4}\left(\alpha_{e}+\gamma_{e i}\right)^{2}\right]\left\|\left(\mathbb{M}_{i}^{\varepsilon}\right)^{\frac{1}{2}} \mathbb{E}_{i}^{n}\right\|^{2}+} \\
& \frac{\Delta t^{2}}{4} \gamma_{e i}\left(\alpha_{e}+\gamma_{e i}\right)\left\|\left(\mathbb{M}_{e}^{\varepsilon}\right)^{\frac{1}{2}} \mathbb{E}_{e}^{n}\right\|^{2}+ \\
& \frac{\Delta t^{2}}{4} \alpha_{e}\left(\alpha_{e}+\gamma_{e i}\right)\left\|\left(\mathbb{M}_{i}^{\varepsilon}\right)^{\frac{1}{2}} \mathbb{E}_{i}^{n}\right\|^{2}+ \\
& \left\|\left(\mathbb{M}_{e}^{\mu}\right)^{\frac{1}{2}} \mathbb{H}_{e}^{n}\right\|^{2}+\left\|\left(\mathbb{M}_{i}^{\mu}\right)^{\frac{1}{2}} \mathbb{H}_{i}^{n}\right\|^{2} .
\end{aligned}
$$

Finally, the hybrid part of the discrete electromagnetic energy (14) yields:

$$
\begin{aligned}
\mathcal{E}_{h}^{n}=-\frac{\Delta t^{2}}{4}{ }^{t} \mathbb{H}_{i}^{n}\left(\mathbb{M}_{i}^{\mu}\right)^{\frac{1}{2}}\left(\mathbb{M}_{i}^{\mu}\right)^{-\frac{1}{2} t} \mathbb{A}_{e i}\left(\mathbb{M}_{e}^{\varepsilon}\right)^{-\frac{1}{2}} \\
\left(\mathbb{M}_{e}^{\varepsilon}\right)^{-\frac{1}{2}} \mathbb{A}_{e i}\left(\mathbb{M}_{i}^{\mu}\right)^{-\frac{1}{2}}\left(\mathbb{M}_{i}^{\mu}\right)^{\frac{1}{2}} \mathbb{H}_{i}^{n} \\
\geq-\frac{\Delta t^{2}}{4}\left\|\left(\mathbb{M}_{e}^{\varepsilon}\right)^{-\frac{1}{2}} \mathbb{A}_{e i}\left(\mathbb{M}_{i}^{\mu}\right)^{-\frac{1}{2}}\right\|^{2}\left\|\left(\mathbb{M}_{i}^{\mu}\right)^{\frac{1}{2}} \mathbb{H}_{i}^{n}\right\|^{2} .
\end{aligned}
$$

Gathering (21) and (22) leads to: 


$$
\begin{aligned}
\mathcal{E}^{n}=\mathcal{E}_{e}^{n}+\mathcal{E}_{i}^{n}+\mathcal{E}_{h}^{n} \geq & {\left[1-\frac{\Delta t^{2}}{4}\left(\alpha_{e}+\gamma_{e i}\right)^{2}\right]\left\|\left(\mathbb{M}_{e}^{\varepsilon}\right)^{\frac{1}{2}} \mathbb{E}_{e}^{n}\right\|^{2}+} \\
& {\left[1-\frac{\Delta t^{2}}{4}\left(\alpha_{e}+\gamma_{e i}\right)^{2}\right]\left\|\left(\mathbb{M}_{i}^{\varepsilon}\right)^{\frac{1}{2}} \mathbb{E}_{i}^{n}\right\|^{2}+} \\
& {\left[1-\frac{\Delta t^{2}}{4} \beta_{e i}^{2}\right]\left\|\left(\mathbb{M}_{i}^{\mu}\right)^{\frac{1}{2}} \mathbb{H}_{i}^{n}\right\|^{2}+} \\
& \frac{\Delta t^{2}}{4} \gamma_{e i}\left(\alpha_{e}+\gamma_{e i}\right)\left\|\left(\mathbb{M}_{e}^{\varepsilon}\right)^{\frac{1}{2}} \mathbb{E}_{e}^{n}\right\|^{2}+ \\
& \frac{\Delta t^{2}}{4} \alpha_{e}\left(\alpha_{e}+\gamma_{e i}\right)\left\|\left(\mathbb{M}_{i}^{\varepsilon}\right)^{\frac{1}{2}} \mathbb{E}_{i}^{n}\right\|^{2}+ \\
& \left\|\left(\mathbb{M}_{e}^{\mu}\right)^{\frac{1}{2}} \mathbb{H}_{e}^{n}\right\|^{2}+\left\|\left(\mathbb{M}_{i}^{\mu}\right)^{\frac{1}{2}} \mathbb{H}_{i}^{n}\right\|^{2} .
\end{aligned}
$$

Finally, using the relation:

$$
1-\frac{\Delta t^{2}}{4} \beta_{e i}^{2}=1-\frac{\Delta t^{2}}{4}\left(\alpha_{e}+\beta_{e i}\right)^{2}+\left(\alpha_{e}^{2}+2 \alpha_{e} \beta_{e i}\right),
$$

allows to obtain that under the conditions:

$$
\Delta t \leq \frac{2}{\alpha_{e}+\gamma_{e i}} \text { and } \Delta t \leq \frac{2}{\alpha_{e}+\beta_{e i}},
$$

that is:

$$
\Delta t \leq \frac{2}{\max \left(\alpha_{e}+\gamma_{e i}, \alpha_{e}+\beta_{e i}\right)}=\frac{2}{\alpha_{e}+\max \left(\gamma_{e i}, \beta_{e i}\right)},
$$

$\mathcal{E}^{n}$ is a positive definite quadratic form of the numerical unknowns $\mathbb{E}_{e}^{n}, \mathbb{E}_{i}^{n}, \mathbb{H}_{e}^{n}$ and $\mathbb{H}_{i}^{n}$ and (15) states a sufficient condition for the stability of the hybrid explicit-implicit DGTD- $\mathbb{P}_{p}$ method (13).

In summary, (15) states that the stability of the hybrid explicit-implicit DGTD- $\mathbb{P}_{p}$ method is deduced from a criterion which is essentially the one obtained for the fully explicit method here restricted to the subset of explicit elements $\mathcal{S}_{e}$, augmented by two terms involving elements of the implicit subset $\mathcal{S}_{i}$ associated to hybrid internal interfaces (i.e. interfaces $a_{i k}$ such that $\tau_{i} \in \mathcal{S}_{e}$ and $\tau_{k} \in \mathcal{S}_{i}$ ). We note that when $\mathcal{S}_{i}=\emptyset$ we get back the condition (10) obtained for the fully explicit scheme. Moreover, if the parameters $\varepsilon$ and $\mu$ are piecewise constant then we have that:

$$
\mathbb{M}_{e, i}^{\varepsilon}=D_{\varepsilon} \mathbb{M}_{e, i} \text { and } \mathbb{M}_{e, i}^{\mu}=D_{\mu} \mathbb{M}_{e, i}
$$

where $D_{\varepsilon}$ and $D_{\mu}$ are diagonal matrices whose entries are the elementwise values $\varepsilon_{i}$ and $\mu_{i}$ respectively, and:

RR $n^{\circ} 6990$ 


$$
\alpha_{e}=\left\|D_{c}\right\|\left\|\left(\mathbb{M}_{e}\right)^{-\frac{1}{2}} \mathbb{S}_{e}\left(\mathbb{M}_{e}\right)^{-\frac{1}{2}}\right\| \quad, \quad \beta_{e i}=\gamma_{e i}=\left\|D_{c}\right\|\left\|\left(\mathbb{M}_{e}\right)^{-\frac{1}{2}} \mathbb{A}_{e i}\left(\mathbb{M}_{i}\right)^{-\frac{1}{2}}\right\|,
$$

where $D_{c}$ denotes the diagonal matrix whose entries are the elementwise values of the propagation speed $c_{i}=1 / \sqrt{\epsilon_{i} \mu_{i}}$. We have that $\left\|D_{c}\right\|$ is equal to $c^{\max }=\max \left(c_{i}\right)$ i.e. to the spectral radii of $D_{c}$. In that case, the stability condition writes:

$$
c^{\max } \Delta t \leq \frac{2}{\alpha_{e}+\beta_{e i}} \leq \frac{2}{\alpha_{e}},
$$

which shows that the stability of the explicit part of the algorithm is guaranteed.

In [FLLP05], the following local condition:

$$
\forall j, \forall k \in \mathcal{V}_{j}, \quad c_{j} \Delta t\left(2 \alpha_{j}+\beta_{j k} \max \left(\sqrt{\frac{\mu_{j}}{\mu_{k}}}, \sqrt{\frac{\varepsilon_{j}}{\varepsilon_{k}}}\right)\right)<\frac{4 V_{j}}{P j},
$$

is proposed for the computation of the global time step where $V_{i}$ and $P_{i}$ respectively denote the volume and the perimeter of element $\tau_{i}$ and where $\alpha_{i}$ and $\beta_{i k}\left(k \in \mathcal{V}_{i}\right)$ are dimensionless constants such that:

$$
\forall \overrightarrow{\mathbf{X}} \in \mathcal{P},\left\{\begin{array}{l}
\|\operatorname{curl} \overrightarrow{\mathbf{X}}\|_{\tau_{i}} \leq \frac{\alpha_{i} P_{i}}{V_{i}}\|\overrightarrow{\mathbf{X}}\|_{\tau_{i}}, \\
\|\overrightarrow{\mathbf{X}}\|_{a_{i k}}^{2} \leq \frac{\beta_{i k} \mathrm{~S}_{i k}}{V_{i}}\|\overrightarrow{\mathbf{X}}\|_{\tau_{i}}^{2}
\end{array}\right.
$$

where and $\|\overrightarrow{\mathbf{X}}\|_{\tau_{i}}$ and $\|\overrightarrow{\mathbf{X}}\|_{a_{i k}}$ denote the $L^{2}$-norms of the vector field $\overrightarrow{\mathbf{X}}$ over $\tau_{i}$ and the interface $a_{i k}$ respectively. From the practical point of view, condition (24) is implemented by looping over the internal interfaces of the mesh $\mathcal{T}_{h}$. Clearly, obtaining the global time step verifying (15) can proceed similarly by applying condition (24) to the explicit internal interfaces (i.e.interfaces $a_{i k}$ such that both $\tau_{i}$ and $\tau_{k}$ belong to $\mathcal{S}_{e}$ ) and hybrid internal interfaces (i.e.interfaces $a_{i k}$ such that $\tau_{i} \in \mathcal{S}_{e}$ and $\tau_{k} \in \mathcal{S}_{i}$ or vice versa).

\section{Numerical assessment of accuracy in 2D}

In this section, the accuracy of the hybrid explicit-implicit DGTD- $\mathbb{P}_{p}$ method is assessed numerically by applying to the solution of the homogeneous source free 2D TM Maxwell equations:

$$
\frac{\partial H_{x}}{\partial t}+\frac{\partial E_{z}}{\partial y}=0, \frac{\partial H_{y}}{\partial t}-\frac{\partial E_{z}}{\partial x}=0, \frac{\partial E_{z}}{\partial t}-\frac{\partial H_{y}}{\partial x}+\frac{\partial H_{x}}{\partial y}=0,
$$

and by considering propagation problems for which an analytical solution is known. 


\subsection{Propagation of an eigenmode in a perfectly conducting cavity}

The first test problem is the propagation of an eigenmode in a unitary square cavity with perfectly conducting walls. Although simple at first glance, this test problem is interesting in the present context because there is no physical mechanism of dissipation of the numerical error and thus it is particularly challenging for assessing the actual accuracy of a given method. The hybrid explicit-implicit DGTD- $\mathbb{P}_{p}$ method is used in conjunction with a nonuniform triangular mesh (see Fig. 1) which consists of 1400 vertices and 2742 triangles. In this case, the minimum and maximum values of the time step are respectively equal to $(\Delta t)_{m}=1.44$ picoseconds and $(\Delta t)_{M}=235.33$ picoseconds (the ratio between the two values $\delta=(\Delta t)_{M} /(\Delta t)_{m}$ is equal to 162$)$.

For this problem, the geometric criterion $c_{g}$ used for the definition of the subsets $\mathcal{S}_{e}$ and $\mathcal{S}_{i}$ is the area of a triangle. The distribution of the element area for the triangular mesh at hand is shown on Fig. 2. From this distribution, several values of $c_{g}$ have been selected for the separation threshold which are summarized in Tab. 1. In this table, $\left|\mathcal{S}_{i}\right|$ and $\left|\mathcal{S}_{e}\right|$ respectively denote the size (in terms of the number of elements) of the subsets $\mathcal{S}_{i}$ and $\mathcal{S}_{e}$. Then, the reference time step $(\Delta t)_{r}$ used for the simulation is the one corresponding to the smallest element of $\mathcal{S}_{e}$ from which we deduce the effective CFL applied to the elements of the implicit subset $\mathcal{S}_{i}$ (denoted by $\mathrm{CFL}_{i}$ in the table). For the purely explicit time scheme, the time step used in the simulations is $\mathrm{CFL}_{e} \times(\Delta t)_{m}$ where $\mathrm{CFL}_{e}$ is the CFL number defining the stability limit of the fully explicit DGTD- $\mathbb{P}_{p}$ method and is respectively equal to 0.2 and 0.3 for $p=1$ and $p=2$.

Results are presented in the form of the time evolution of the L2 error between the approximate and analytic solutions, see Fig. 3 for simulations based on the hybrid explicit-implicit DGTD- $\mathbb{P}_{1}$ and DGTD- $\mathbb{P}_{2}$ methods. On these figures, the corresponding time evolutions for the fully explicit methods are also shown for comparison. Above a certain threshold value for $c_{g}$, the time step applied to the implicit part is responsible for a dispersion error that deteriorates the overall accuracy of the hybrid explicit-implicit DGTD- $\mathbb{P}_{p}$ method. The choice of the optimal time step yielding an error level not exceeding that of the fully explicit DGTD- $\mathbb{P}_{p}$ method is not a trivial task. It requires a detailed analysis of the numerical dispersion error of the hybrid explicit-implicit DGTD- $\mathbb{P}_{p}$ method and the definition of an error estimator allowing the development of an auto-adaptive numerical methodology. These tasks have not been considered so far and are postponed for a future study.

\subsection{Scattering of a plane wave by a dielectric cylinder}

We present here results for the scattering of a plane wave with frequency $\mathrm{F}=300 \mathrm{MHz}$ by a dielectric cylinder. This test problem has been considered in several works such as [CD03] where the expression of the analytical solution is detailed. The internal cylinder has a radius $r_{0}=0.6 \mathrm{~m}$ and bounds a material with relative permittivity $\epsilon_{2}=2.25$. The computational domain $\Omega$ is bounded by a square of side length $a=3.2$ centered at $(0,0)$. A first order Silver-Müller absorbing condition is applied on the boundary of the square. A non-uniform mesh is used which consists of 4108 vertices and 8054 triangles. The minimum and maximum

RR $n^{\circ} 6990$ 


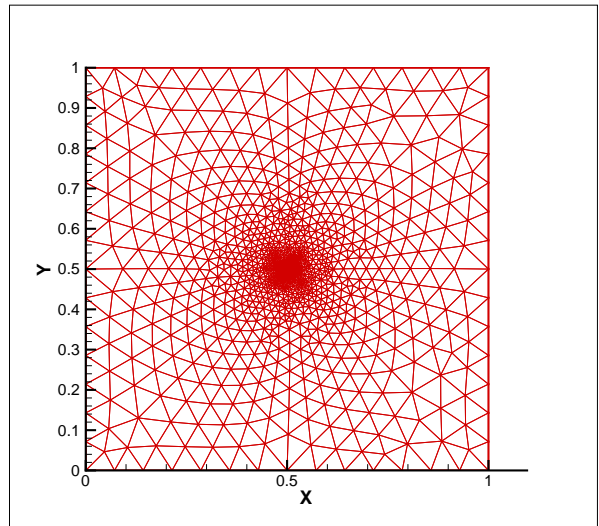

Figure 1: Non-uniform triangular mesh of a unitary square cavity.

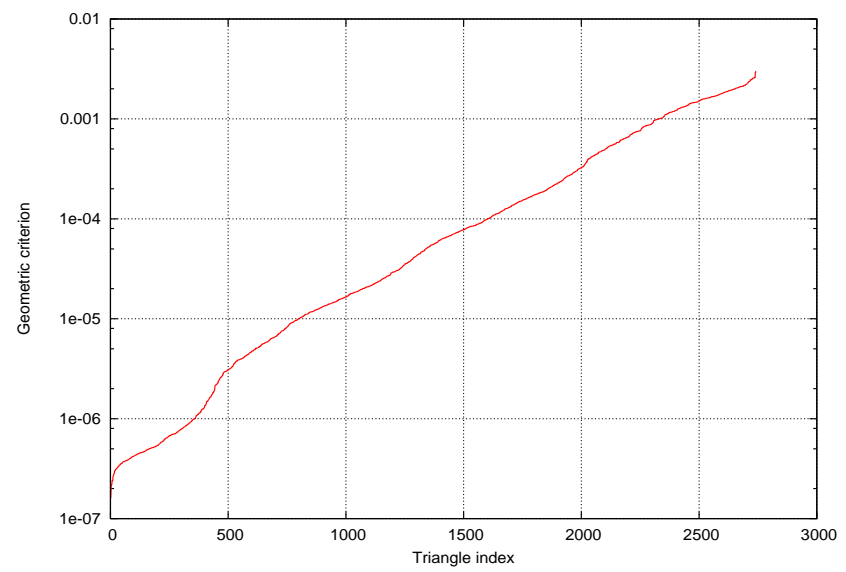

Figure 2: Eigenmode in a unitary square cavity: distribution of the geometric criterion. 

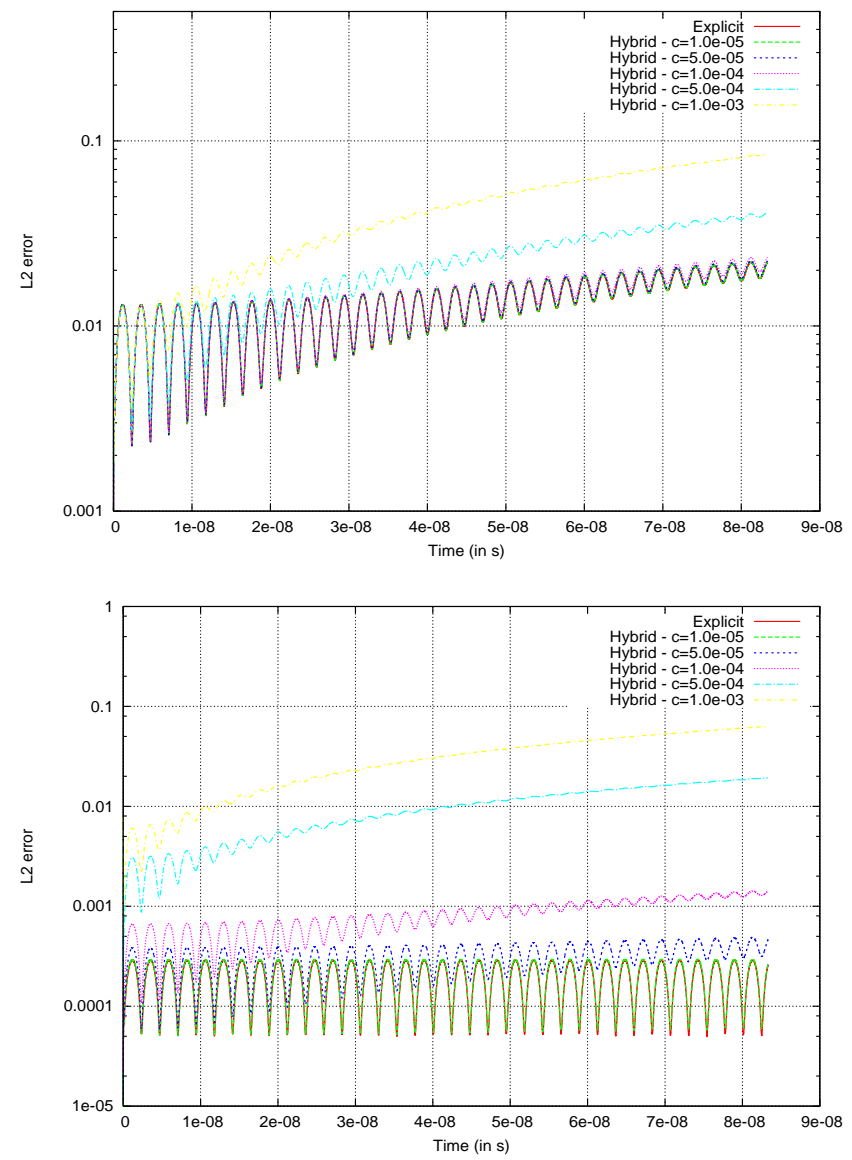

Figure 3: Eigenmode in a unitary square cavity: time evolution of the L2 error. Hybrid explicit-implicit DGTD- $\mathbb{P}_{1}$ method (top) and DGTD- $\mathbb{P}_{2}$ method (bottom). 


\begin{tabular}{c|c|r|r|c|c} 
Method & $c_{g}$ threshold & $\left|\mathcal{S}_{i}\right|$ & $\left|\mathcal{S}_{e}\right|$ & $(\Delta t)_{r}$ (picoseconds) & $\mathrm{CFL}_{i}$ \\
\hline DGTD- $\mathbb{P}_{1}$ & $10^{-5} \mathrm{~m}^{2}$ & 1943 & 799 & 3.18 & 2.2 \\
- & $5.10^{-5} \mathrm{~m}^{2}$ & 1403 & 1339 & 7.28 & 5.0 \\
- & $10^{-4} \mathrm{~m}^{2}$ & 1141 & 1601 & 10.63 & 7.3 \\
- & $5.10^{-4} \mathrm{~m}^{2}$ & 640 & 2102 & 27.39 & 18.9 \\
- & $10^{-3} \mathrm{~m}^{2}$ & 415 & 2327 & 39.36 & 27.2 \\
\hline DGTD- $\mathbb{P}_{2}$ & $10^{-5} \mathrm{~m}^{2}$ & 1943 & 799 & 2.12 & 1.4 \\
- & $5.10^{-5} \mathrm{~m}^{2}$ & 1403 & 1339 & 4.85 & 3.3 \\
- & $10^{-4} \mathrm{~m}^{2}$ & 1141 & 1601 & 7.08 & 4.9 \\
- & $5.10^{-4} \mathrm{~m}^{2}$ & 640 & 2102 & 18.26 & 12.6 \\
- & $10^{-3} \mathrm{~m}^{2}$ & 415 & 2327 & 26.24 & 18.1
\end{tabular}

Table 1: Eigenmode in a unitary square cavity. Simulation configurations for the hybrid explicit-implicit DGTD- $\mathbb{P}_{p}$ method.

values of the renormalized time step are respectively equal to $(\Delta t)_{m}=2.09$ picoseconds and $(\Delta t)_{M}=309.63$ picoseconds (the ratio between the two values $\delta=(\Delta t)_{M} /(\Delta t)_{m}$ is equal to 148). As for the previous problem, the geometric criterion $c_{g}$ used for the definition of the subsets $\mathcal{S}_{e}$ and $\mathcal{S}_{i}$ is the area of a triangle. The distribution of the element area for the triangular mesh at hand is shown on Fig. 4. The minimum and maximum values of the area of a triangle are respectively equal to $0.25 \times 10^{-6} \mathrm{~m}^{2}$ and $0.65 \times 10^{-2} \mathrm{~m}^{2}$. We have selected $10^{-4} \mathrm{~m}^{2}$ as a threshold value for the geometric criterion $c_{g}$ which yields $\left|\mathcal{S}_{e}\right|=5,745$ and $\left|\mathcal{S}_{i}\right|=2,309$. The simulation time has been set to 10 periods of the incident wave.

Contour lines of $E_{z}$ for the analytical solution and the solution resulting from the hybrid explicit-implicit DGTD- $\mathbb{P}_{2}$ method are shown on Fig. 5. On Fig. 6 we compare the $x$-wise distributions for $y=0$ of the real part of the discrete Fourier transform of $E_{z}$ (denoted as $\operatorname{DFT}\left(E_{z}\right)$ ) for the approximate solutions obtained using the explicit, implicit and hybrid explicit-implicit DGTD- $\mathbb{P}_{1}$ and DGTD- $\mathbb{P}_{2}$ methods. Finally, a comparison in terms of the time evolution of the L2 error is also shown on Fig. 7. Performance figures are gathered in Tab. 2 for simulations conducted on a workstation equipped with AMD Opteron $2 \mathrm{GHz}$ prcessor and 2 GB of RAM memory. In this table, the reported CFL values are those used for the explicit, implicit and hybrid explicit-implicit methods where in the latter case we mention the values applied to the elements of the subsets $\mathcal{S}_{e}$ and $\mathcal{S}_{i}$. For this test problem and the considered triangular mesh, the hybrid explicit-implicit DGTD- $\mathbb{P}_{1}$ and DGTD- $\mathbb{P}_{2}$ methods allow a reduction of the computational time by a factor 3.3 and yield approximate solutions that are almost coincident with those of their explicit counterparts.

\section{Numerical and performance results in $3 \mathrm{D}$}

In this section we apply the proposed hybrid explicit-implicit DGTD- $\mathbb{P}_{p}$ method to the simulation of realistic 3D electromagnetic wave propagation problems. The implicit system of 


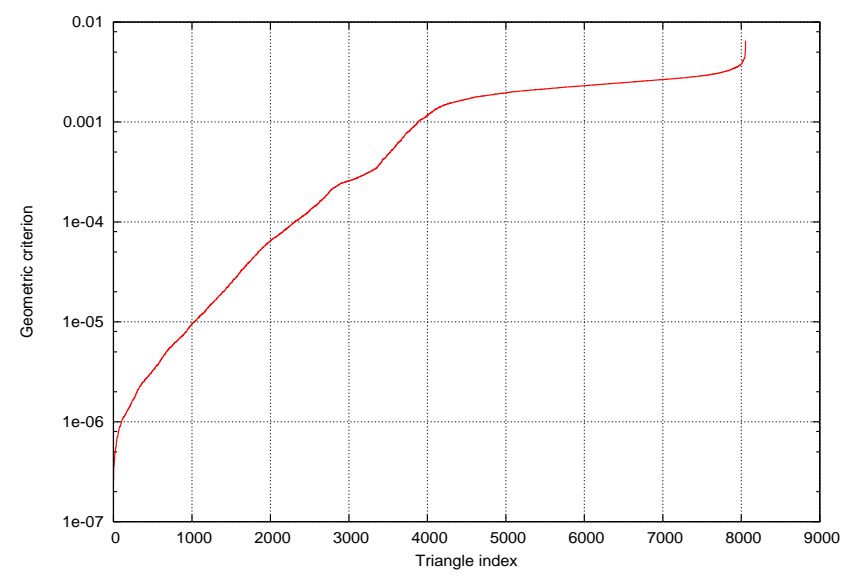

Figure 4: Scattering of a plane wave by a dielectric cylinder: distribution of the geometric criterion.

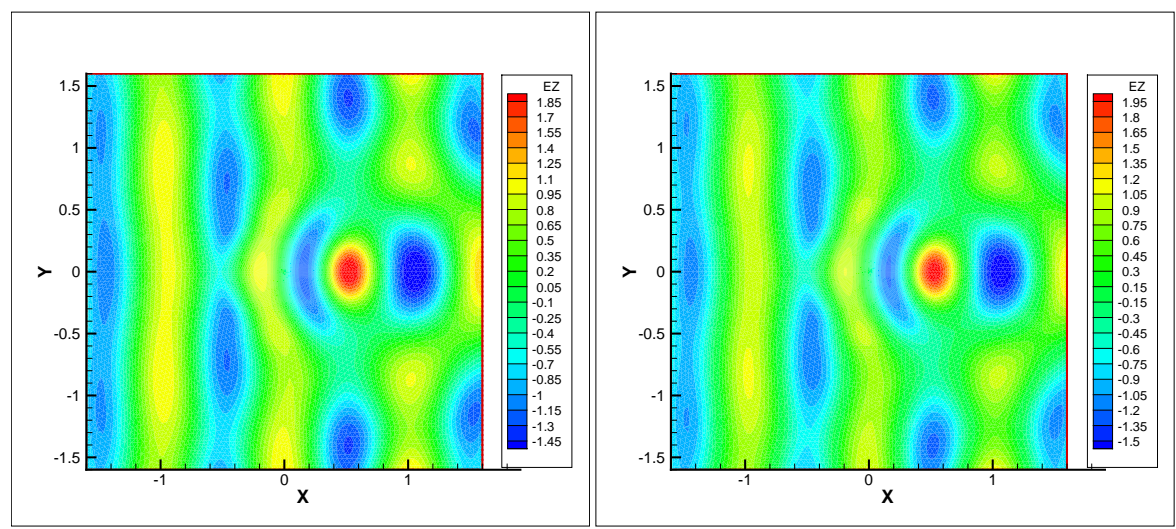

Figure 5: Scattering of a plane wave by a dielectric cylinder. Contour lines of $E_{z}$ after 10 periods. Analytical solution (left) and hybrid explicit-implicit DGTD- $\mathbb{P}_{2}$ method (right). 

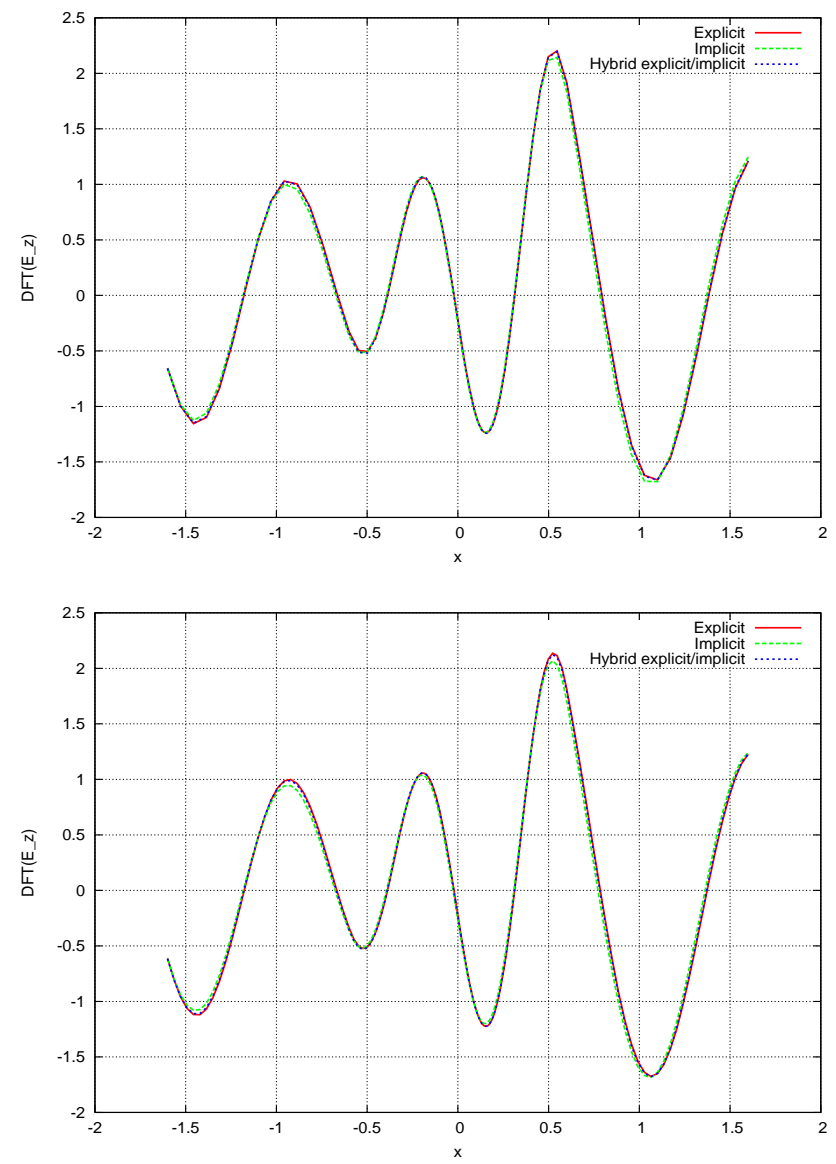

Figure 6: Scattering of a plane wave by a dielectric cylinder: $x$-wise distribution of $\operatorname{DFT}\left(E_{z}\right)$. DGTD- $\mathbb{P}_{1}$ methods (left) and DGTD- $\mathbb{P}_{2}$ methods (right).

\begin{tabular}{c|c|c|c|c} 
Method & Time integration & CFL & Total time & Gain \\
\hline DGTD- $\mathbb{P}_{1}$ & Explicit & 0.3 & $477 \mathrm{sec}$ & - \\
- & Implicit & 21.0 & $109 \mathrm{sec}$ & 4.4 \\
- & Hybrid explicit-implicit & $0.3 / 17.5$ & $145 \mathrm{sec}$ & 3.3 \\
\hline DGTD- $\mathbb{P}_{2}$ & Explicit & 0.2 & $1805 \mathrm{sec}$ & - \\
- & Implicit & 21.0 & $257 \mathrm{sec}$ & 7.0 \\
- & Hybrid explicit-implicit & $0.2 / 17.5$ & $524 \mathrm{sec}$ & 3.4
\end{tabular}

Table 2: Scattering of a plane wave by a dielectric cylinder. Performance results for the explicit, implicit and explicit-implicit DGTD- $\mathbb{P}_{p}$ method. 

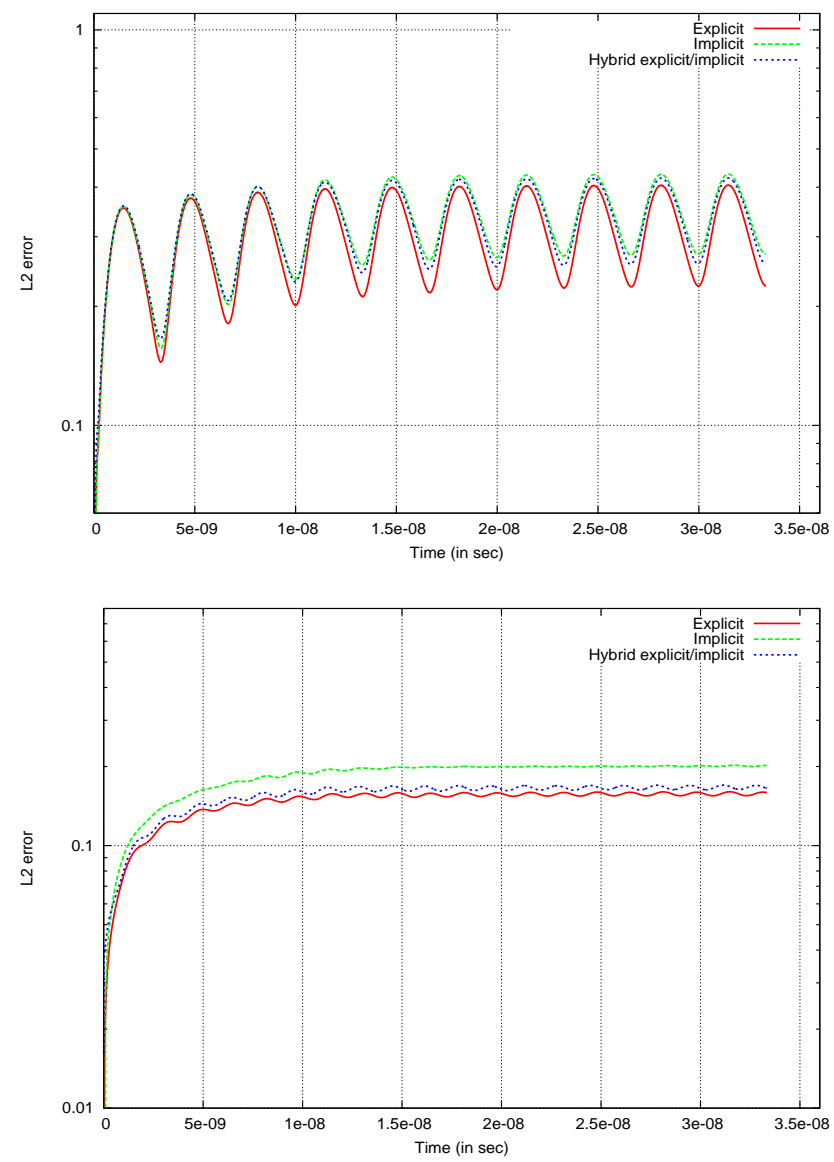

Figure 7: Scattering of a plane wave by a dielectric cylinder: time evolution of the L2 error. DGTD- $\mathbb{P}_{1}$ methods (left) and DGTD- $\mathbb{P}_{2}$ methods (right). 
equations associated to the mesh elements in the subset $\mathcal{S}_{i}$ is solved using the MUMPS optimized sparse direct solver [ADL00]. In the tables of this section, «RAM (LU)»is the memory overhead for computing and storing the sparse L and U factors, while «Time (LU)»gives the factors construction time. Moreover, the geometric criterion used for the definition of the subsets $\mathcal{S}_{i}$ and $\mathcal{S}_{e}$ is chosen to be:

$$
c_{g}\left(\tau_{i}\right)=4 \min _{j \in \mathcal{V}_{i}} \frac{V_{i} V_{j}}{P_{i} P_{j}},
$$

where $V_{i}$ and $P_{i}$ respectively denote the volume and the perimeter of the tetrahedron $\tau_{i}$ and where we recall that $\mathcal{V}_{i}=\left\{j \mid \tau_{i} \cap \tau_{j} \neq 0\right\}$.

\subsection{Scattering of a plane wave by an aircraft}

The problem under consideration is the simulation of the scattering of a plane wave by an aircraft geometry. The frequency of the incident field is $\mathrm{F}=200 \mathrm{MHz}$ (the wavelength is $\lambda=1.5 \mathrm{~m}$ ). The computational domain is artificially bounded by a parallelepipedic box on which the Silver-Müller condition is imposed. The underlying tetrahedral mesh consists of 360,495 vertices and 2,024,924 tetrahedra. The minimum and maximum lengths of the mesh edges are respectively equal to $9.1610^{-3} \mathrm{~m}$ (which corresponds to $\approx \lambda / 163 \mathrm{~m}$ ) and $6.8310^{-1} \mathrm{~m}$ (which corresponds to $\approx \lambda / 2.2 \mathrm{~m}$ ). The minimum and maximum values of the time step are respectively equal to $(\Delta t)_{m}=7.7$ picoseconds and $(\Delta t)_{M}=444.3$ picoseconds (the ratio $\left.\delta=(\Delta t)_{M} /(\Delta t)_{m}=58\right)$. The distribution of the criterion (27) for the tetrahedral mesh at hand is shown on Fig. 9. The simulations discussed here have been performed on a workstation equipped with an Intel Xeon $2.33 \mathrm{GHz}$ processor and $32 \mathrm{~GB}$ of RAM memory. We report on results obtained using the fully explicit and hybrid explicit-implicit DGTD- $\mathbb{P}_{1}$ methods. The contour lines of $|\mathbf{E}|$ on the surface of the aircraft, for a physical simulation time corresponding to 5 periods of the incident wave, are shown on Fig. 8. Time evolutions of the $E_{z}$ component at a selected point are compared on Fig. 10. Performance results for the simulations based on the hybrid explicit-implicit DGTD- $\mathbb{P}_{1}$ method are summarized in Tab. 4. The simulation using the fully explicit DGTD- $\mathbb{P}_{1}$ method gives the reference computing time which is equal to $25 \mathrm{~h} 3 \mathrm{mn}$. The results of Tab. 4 show that the memory overhead associated to the construction and the storage of the $\mathrm{L}$ and $\mathrm{U}$ factors of the implicit matrix is acceptable while the gain in computing time ranges from 3.7 to 6.2 for the considered threshold values of the geometric criterion $c_{g}$.

\begin{tabular}{c|c|c|c}
$c_{g}$ threshold & $\left|\mathcal{S}_{e}\right|$ & $\left|\mathcal{S}_{i}\right|$ & $\mathrm{CFL}_{i}$ \\
\hline 0.0125 & $2,024,320$ & 604 & 1.81 \\
0.0175 & $2,022,464$ & 2460 & 2.53 \\
0.02 & $2,018,543$ & 6381 & 2.90
\end{tabular}

Table 3: Scattering of a plane wave by an aircraft. Definition of the subsets $\mathcal{S}_{e}$ and $\mathcal{S}_{i}$ for different values of $c_{g}$. 


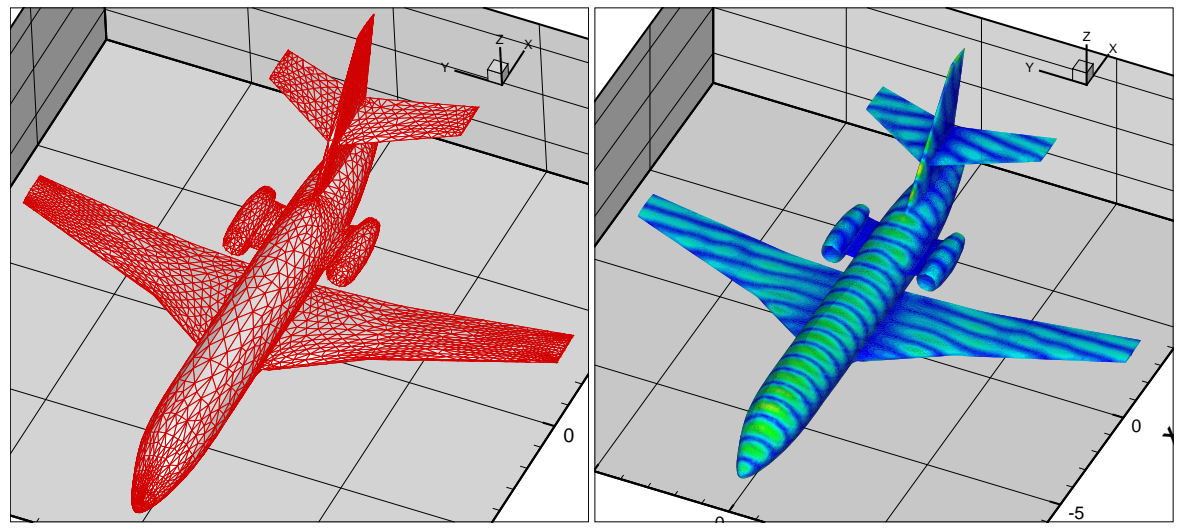

Figure 8: Scattering of a plane wave by an aircraft. Triangulation (left) and contour lines of $|\mathbf{E}|$ (right) on the aircraft surface.

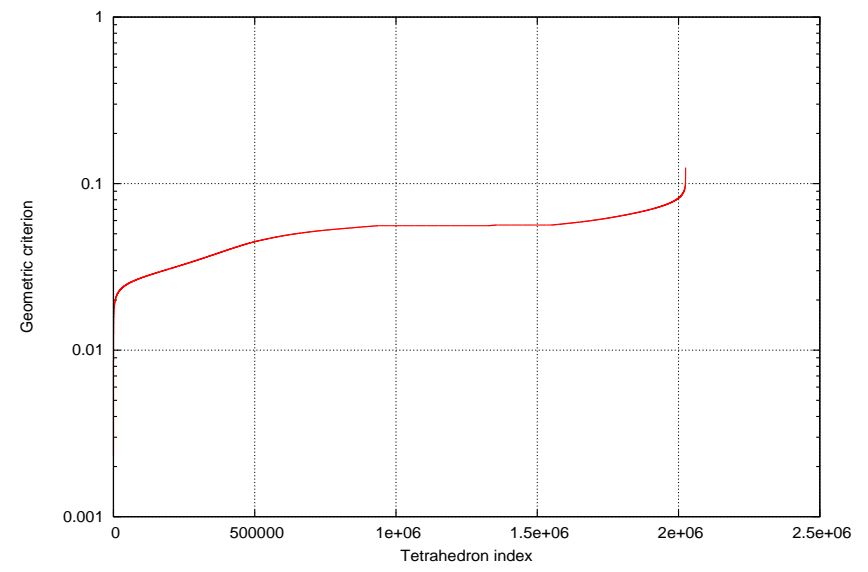

Figure 9: Scattering of a plane wave by an aircraft: distribution of the geometric criterion.

\begin{tabular}{c|c|c|c}
$c_{g}$ threshold & RAM $(\mathrm{LU})$ & Time $(\mathrm{LU})$ & Total time \\
\hline $0.0125 \mathrm{~m}$ & $12 \mathrm{MB}$ & $0.3 \mathrm{sec}$ & $6 \mathrm{~h} 39 \mathrm{mn}$ \\
$0.0175 \mathrm{~m}$ & $48 \mathrm{MB}$ & $1.5 \mathrm{sec}$ & $4 \mathrm{~h} 44 \mathrm{mn}$ \\
$0.02 \mathrm{~m}$ & $117 \mathrm{MB}$ & $4.2 \mathrm{sec}$ & $4 \mathrm{~h} 08 \mathrm{mn}$
\end{tabular}

Table 4: Scattering of a plane wave by an aircraft. Performance results for the hybrid explicit-implicit DGTD- $\mathbb{P}_{1}$ method. Reference time for the fully explicit DGTD- $\mathbb{P}_{1}$ method : $25 \mathrm{~h} 3 \mathrm{mn}$. 


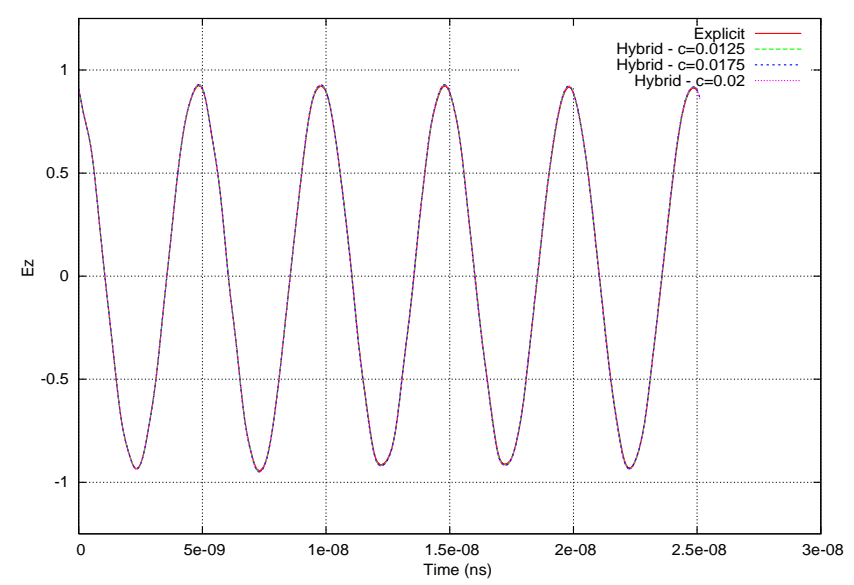

Figure 10: Scattering of a plane wave by an aircraft. Time evolution of the $E_{z}$ component at a selected point.

\subsection{Exposure of head tissues to a localized source radiation}

We now consider a more realistic problem which consists in the simulation of the exposure of a geometrical model of head tissues to an electromagnetic wave emitted by a localized source. Starting from MR images of the Visible Human project [RHGJ03], head tissues are segmented and the interfaces of a selected number of tissues (namely, the skin, the skull and the brain) are triangulated. Different strategies can be used in order to obtain a smooth and accurate segmentation of head tissues and associated interface triangulations. A first strategy consists in using a marching cube algorithm [LC87] which leads to huge triangulations of interfaces between segmented subdomains. These triangulations can then be regularized, refined and decimated in order to obtain reasonable surface meshes, for example using the YAMS [Fre03] re-meshing tool. Another strategy consists in using a variant of Chew's algorithm [Che93], based on Delaunay triangulation restricted to the interface, which allows to control the size and aspect ratio of interface triangles [BO05]. Surface meshes of the skin, skull and brain resulting from such a procedure are presented on Fig. 11. Then, these triangulated surfaces are used as inputs for the generation of volume meshes. In this study, the GHS3D tetrahedral mesh generator [GHS91] is used to mesh volume domains between the various interfaces. Note that the exterior of the head must also be meshed, up to a certain distance from the skin. The computational domain is here artificially bounded by a sphere on which the Silver-Müller condition is imposed. Moreover, a simplified mobile phone model is included and placed in vertical position close to the right ear (see Fig. 12).

In the present case, the constructed geometrical model consists of four tissues (skin, skull, CSF - Cerebro Spinal Fluid, brain) and the global tetrahedral mesh consists of 316,172 
vertices and 1,889,982 tetrahedra. The minimum and maximum lengths of the mesh edges are respectively equal to $0.0625 \mathrm{~mm}$ and $23.814 \mathrm{~mm}$ (in the vacuum zone). The smallest elements are located in the skin depth on one hand, and between the bottom surface of the antenna and the top surface of the metallic box on the other hand. The minimum and maximum values of the time step are respectively equal to $(\Delta t)_{m}=0.023$ picoseconds and $(\Delta t)_{M}=16.02$ picoseconds (the ratio $\delta=(\Delta t)_{M} /(\Delta t)_{m}=696$ ). The distribution of the criterion (27) for the tetrahedral mesh at hand is shown on Fig. 13. The characteristics of the tissues are summarized in Tab. 5 where the values of the electrical permittivity correspond to a frequency $\mathrm{F}=1800 \mathrm{MHz}$ and have been obtained from a special purpose online data base. We assume here that the tissues are non-conducting although it is clear that for a more realistic modeling of the propagation, conductivity should also be taken into account. Finally, a dipolar type source is localized between the bottom surface of the antenna and the top surface of the metallic box yielding a current of the form $\left(\mathbf{x}_{d}\right.$ is the localization point of the source):

$$
J_{z}(\mathbf{x}, t)=\delta\left(\mathbf{x}-\mathbf{x}_{d}\right) \sin (\omega t)
$$

which is discretized according to the discontinuous Galerkin formulation discussed in section 3 .

Numerical simulations have been conducted on a cluster of 20 Intel Xeon $2.33 \mathrm{GHz}$ based nodes interconnected by a high performance Myrinet network. Each node consists of a dual processor quad core board sharing $16 \mathrm{~GB}$ of RAM memory. We report on results obtained using the fully explicit DGTD- $\mathbb{P}_{1}$ method and the hybrid explicit-implicit DGTD- $\mathbb{P}_{1}$ and DGTD- $\mathbb{P}_{2}$ methods. A single value of the criterion (27) has been considered for the definition of the subsets $\mathcal{S}_{i}$ and $\mathcal{S}_{e}$ (see Tab. 6). For this selected threshold value, only $1.7 \%$ of the mesh elements are treated implicitly. The physical simulation time has been fixed to 6 periods of the temporal signal of (28). A discrete Fourier transform of the components of the electric field is computed during the last period of the simulation. Contour lines of the module of the real part of the discrete Fourier transform of $\mathbf{E}$ (denoted by $\mathcal{R}(\operatorname{DFT}(\mathbf{E})$ ) on the skin, skull and brain surfaces for the approximate solutions resulting from the hybrid explicit-implicit DGTD- $\mathbb{P}_{1}$ and DGTD- $\mathbb{P}_{2}$ methods are shown on Fig. 14. Time evolutions of the $E_{z}$ component at two selected points in the free space near the ear and in the brain are compared on Fig. 15. One can note on this figure that the approximate solutions resulting from the fully explicit and hybrid explicit-implicit methods are almost indistinguishable. The simulation using the fully explicit DGTD- $\mathbb{P}_{1}$ method has been conducted on $N_{s}=64$ cores of the above-mentioned cluster for a total computing time of $38 \mathrm{~h} 43 \mathrm{mn}$. Performance results for the simulations based on the hybrid explicit-implicit DGTD- $\mathbb{P}_{1}$ and DGTD- $\mathbb{P}_{2}$ methods are summarized in Tab. 7. Note that these simulations have been performed on 16 and 32 cores. For this problem, the hybrid explicit-implicit DGTD- $\mathbb{P}_{1}$ method allows a reduction of the computing time by a factor of 56 if with assume a linear parallel speedup for the fully explicit DGTD- $\mathbb{P}_{1}$ method to evaluate a reference time on 16 cores. Such an assumption is reasonable for the fully explicit method but dose not apply to the hybrid explicit-implicit method as can be seen in Tab. 7 in the case of the DGTD- $\mathbb{P}_{2}$ method. The 
observed suboptimal parallel speedup is probably due to the use of sparse direct solver for the implicit system.
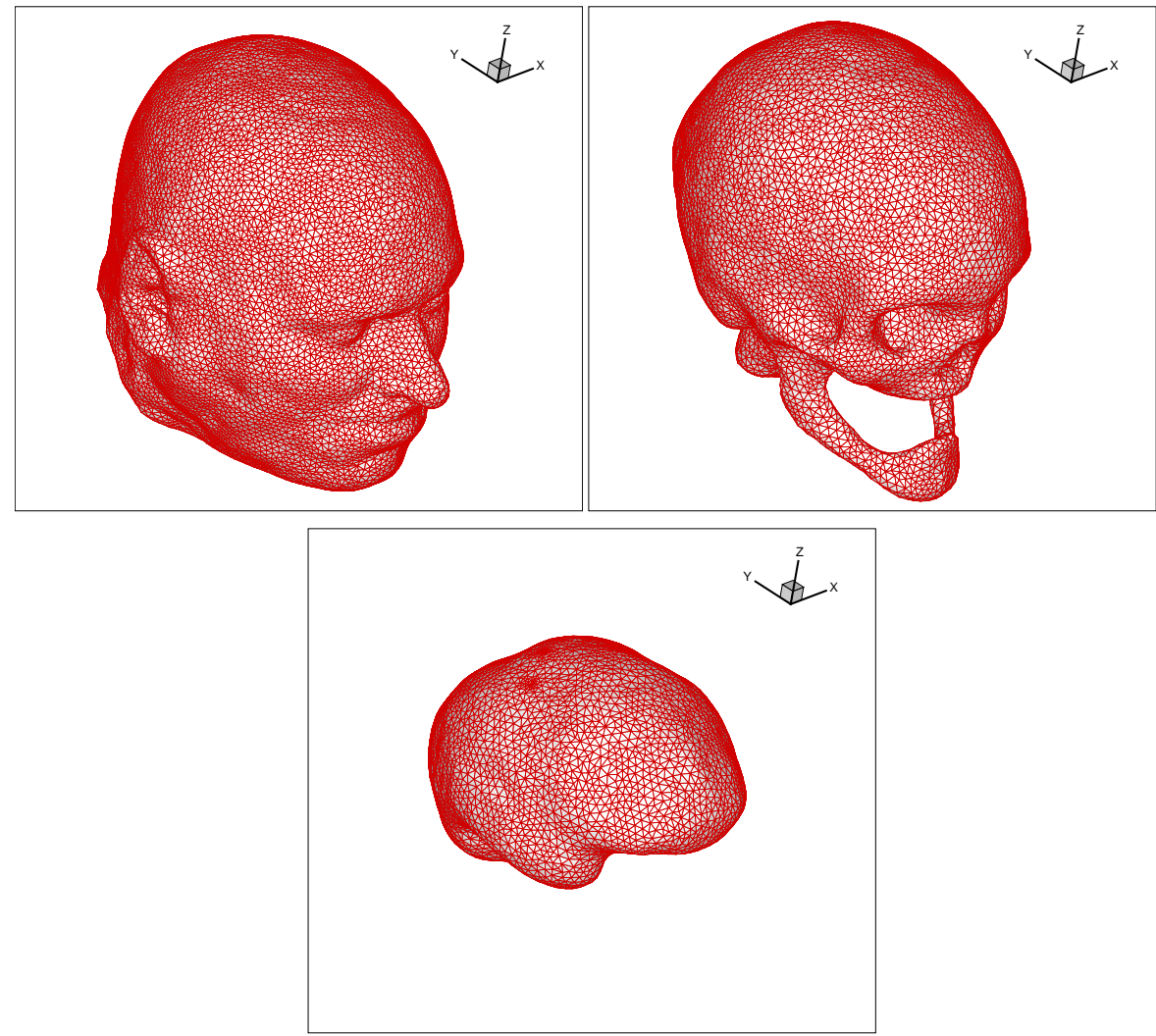

Figure 11: Exposure of head tissues to a localized source radiation: surface meshes of the skin, skull and brain.

\begin{tabular}{c|c|c} 
Tissue & $\varepsilon_{r}$ & $\lambda(\mathrm{mm})$ \\
\hline Vaccum & 1.00 & 166.66 \\
Skin & 43.85 & 26.73 \\
Skull & 15.56 & 42.25 \\
CSF & 67.20 & 20.33 \\
Brain & 43.55 & 25.26
\end{tabular}

Table 5: Exposure of head tissues to a localized source radiation: electromagnetic characteristics of tissues. 


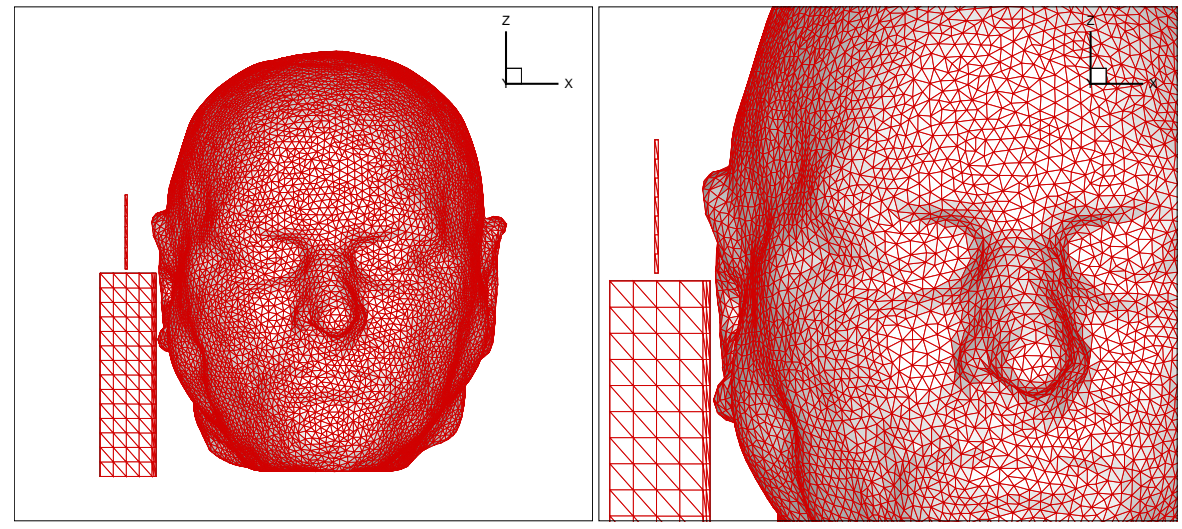

Figure 12: Exposure of head tissues to a localized source radiation: simplified mobile phone model.

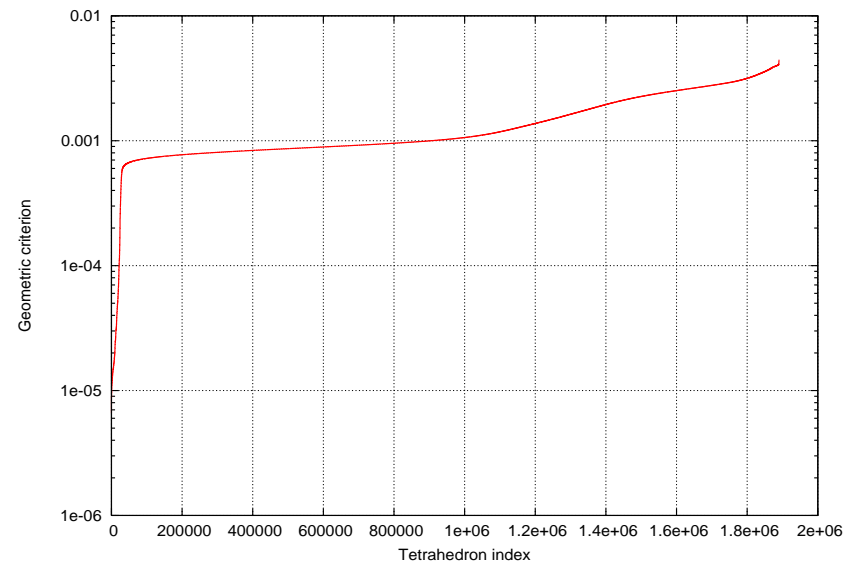

Figure 13: Exposure of head tissues to a localized source radiation: distribution of the geometric criterion.

\begin{tabular}{c|c|c|c}
$c_{g}$ threshold & $\left|\mathcal{S}_{e}\right|$ & $\left|\mathcal{S}_{i}\right|$ & $\mathrm{CFL}_{i}$ \\
\hline $0.0006 \mathrm{~m}$ & $1,858,469$ & 31,513 & $27.24\left(\mathbb{P}_{1}\right) / 15.13\left(\mathbb{P}_{2}\right)$
\end{tabular}

Table 6: Exposure of head tissues to a localized source radiation. Definition of the subsets $\mathcal{S}_{e}$ and $\mathcal{S}_{i}$.

$\mathrm{RR} \mathrm{n}^{\circ} 6990$ 

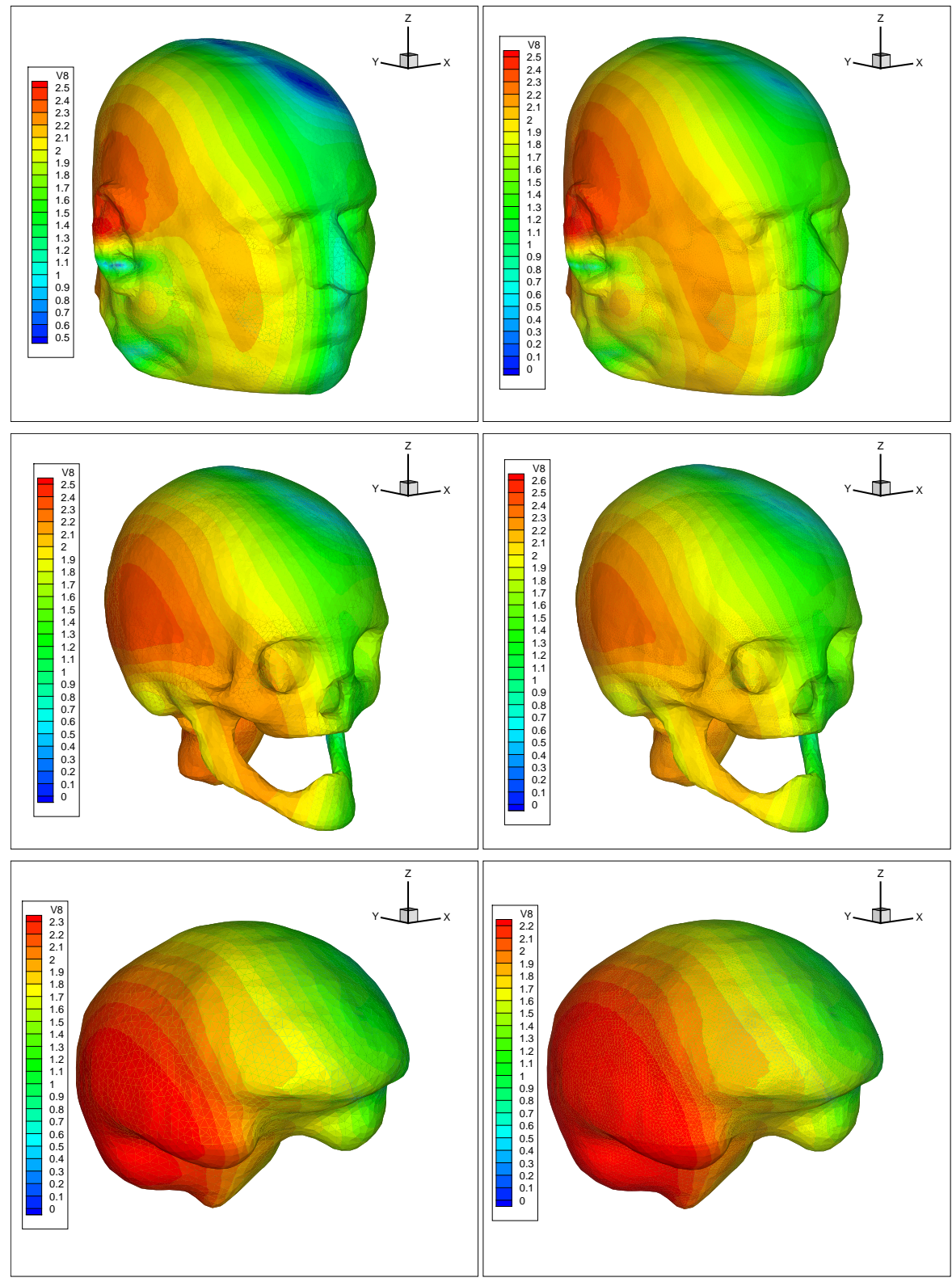

Figure 14: Exposure of head tissues to a localized source radiation. Contour lines of $|\mathcal{R}(\mathbf{E})|$ (in log scale) on the skin, skull and brain surfaces: hybrid explicit-implicit DGTD- $\mathbb{P}_{1}$ (left) and DGTD- $\mathbb{P}_{2}$ (right) methods. 

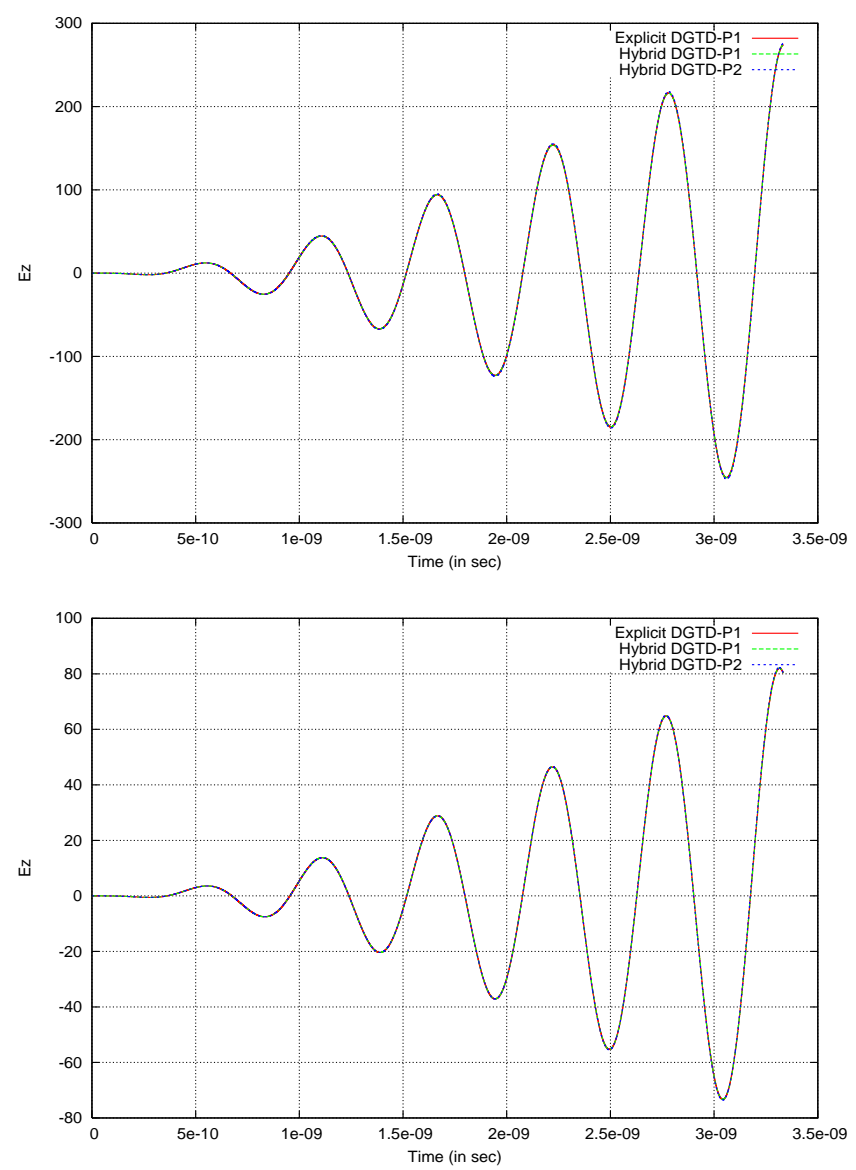

Figure 15: Exposure of head tissues to a localized source radiation. Time evolution of the $E_{z}$ component at selected points in free space (top) and in the brain (bottom).

\begin{tabular}{c|c|c|c|c} 
Method & $N_{s}$ & RAM (LU) & Time (LU) & Total time \\
\hline DGTD- $_{1}$ & 16 & $1.2 \mathrm{~GB}$ & $89.0 \mathrm{sec}$ & $2 \mathrm{~h} 40 \mathrm{mn}$ \\
\hline DGTD- $_{2}$ & 16 & $3.7 \mathrm{~GB}$ & $696.0 \mathrm{sec}$ & $13 \mathrm{~h} 45 \mathrm{mn}$ \\
& 32 & $2.2 \mathrm{~GB}$ & $598.0 \mathrm{sec}$ & $8 \mathrm{~h} 42 \mathrm{mn}$
\end{tabular}

Table 7: Exposure of head tissues to a localized source radiation. Performance results for the hybrid explicit-implicit DGTD- $\mathbb{P}_{i}$ method. Reference time for the fully explicit DGTD- $\mathbb{P}_{1}$ method on $N_{s}=64$ cores : $38 \mathrm{~h} 43 \mathrm{mn}$. 


\section{Conclusion}

We have presented the results of a study aiming at overcoming the grid-induced stiffness in high order DGTD methods formulated on non-uniform simplicial meshes for the solution of the system of time domain Maxwell equations. For that purpose, we have adopted an hybrid explicit-implicit time integration strategy. First, the elements of the underlying mesh are assumed to be partitioned into two sets according to an appropriate geometric criterion. Then, the time integration method considered here combines an implicit CrankNicolson scheme applied to the semi-discretized equations associated to the mesh elements belonging to one of the subsets (typically, the elements localized in the refined zones of the mesh), with an explicit Leap-Frog scheme applied to the semi-discretized equations for the elements in the complementary subset. The resulting strategy is a component splitting based CNLF (Crank-Nicolson-Leap-Frog) DGTD method following the terminology adopted in [Ver09]. A stability analysis of the hybrid explicit-implicit DGTD method using energetic considerations shows that the reference (global) time step can be computed from a condition essentially ensuring that the Leap-Frog scheme alone is stable for the mesh elements of the corresponding subset. The temporal convergence of the CNLF time integration scheme has recently been studied in [Ver09] where a convergence condition is derived that guarantees second order temporal convergence uniformly in the spatial discretization parameter. The accuracy of the CNLF DGTD method has been assessed here numerically in the twodimensional case by considering wave propagation problems for which analytical solutions are available.

Numerical simulations of realistic three-dimensional propagation problems involving locally refined tetrahedral meshes have demonstrated that the proposed CNLF DGTD method allows to reduce significantly the overall computing time as compared to a fully explicit DGTD method, and as long as a rather small number of the mesh elements are treated implicitly (typically a few percent) which is often the case in practical situations involving irregularly shaped objects and material interfaces. However, despite these encouraging results, several points still deserve to be addressed in order to obtain a more accurate and more efficient solution strategy:

- the temporal accuracy could be improved by studying the possibility of combining a high order Leap-Frog scheme [SSW02] with a higher order implicit scheme [LF04], or by considering alternative hybrid explicit-implicit time integration strategies such as those studied in [CS08].

- In all the numerical experiments discussed in this paper, the threshold value for the geometric criterion $c_{g}$ used for the definition of the subsets $\mathcal{S}_{e}$ and $\mathcal{S}_{i}$ has been set statically. This threshold value impacts both the accuracy (more precisely, the numerical dispersion) of the CNLF DGTD method and its overall computational cost (computing time and memory overhead). Clearly, obtaining an auto-adaptive solution strategy which optimizes both aspects would be an asset. In particular, the reference (global) time step ensuring the stability of the method could be adapted dynamically 
in order to control the numerical error. The use of embedded time integration schemes can facilitate this task as demonstrated in [KCGH07] in the context of explicit-implicit Runge-Kutta (IMEX-RK) methods.

- Parallel computing is a mandatory path for large-scale 3D simulations and is well mastered for fully explicit solution methods for the time domain Maxwell equations. However, the parallelization of hybrid explicit-implicit methods such as the CNLF DGTD method considered here raises (at least) two difficulties that have not been addressed in this paper. On one hand, the separation of mesh elements into two subsets induces load balancing issues. Indeed, since the explicit-implicit time integration scheme is a sequential process (see algorithm (13)), the partitioning of the mesh should not simply aims at balancing the mesh elements taking into account a single weight related to the local computational load (i.e.explicit or implicit element). Instead, an appropriate two-constraint partitioning problem aiming at balancing both the explicit and implicit elements should be formulated. On the other hand, a scalable solver must be considered for the linear system associated to the implicit elements.

\section{References}

[ADL00] P.R. Amestoy, I.S. Duff, and J.-Y. L'Excellent, Multifrontal parallel distributed symmetric and unsymmetric solvers, Comput. Meth. App. Mech. Engng. 184 (2000), 501-520.

[BO05] J.-D. Boissonnat and S. Oudot, Provably good sampling and meshing of surfaces, Graphical Models 67 (2005), no. 5, 405-451.

[CCR05] M.H. Chen, B. Cockburn, and F. Reitich, High-order RKDG methods for computational electromagnetics, J. Sci. Comput. 22-23 (2005), 205-226.

[CD03] W. Cai and S. Deng, An upwinding embedded boundary method for Maxwell's equations in media with material interfaces: $2 D$ case, J. Comput. Phys. 190 (2003), no. 1, 159-183.

[CDL09] A. Catella, V. Dolean, and S. Lanteri, An implicit discontinuous Galerkin timedomain method for two-dimensional electromagnetic wave propagation, COMPEL (2009), to appear.

[CFP06] G. Cohen, X. Ferriéres, and S. Pernet, A spatial high order hexahedral discontinuous Galerkin method to solve Maxwell's equations in time-domain, J. Comput. Phys. 217 (2006), no. 2, 340-363.

[Che93] L.P. Chew, Guaranteed-quality mesh generation for curved surfaces, 9th Annual ACM Symposium Computational Geometry, ACM Press, 1993, pp. 274-280. 
[CS08] E.M. Constantinescu and A. Sandu, Achieving very high order for implicit explicit time steppings: extrapolation methods, Tech. Report TR-08-13, Virginia Polytechnic Institute and State University, Computer Science Department, 2008.

[DG07] J. Diaz and M.J. Grote, Energy conserving explicit local time-stepping for secondorder wave equations, Tech. Report 2007-02, Department of Mathematics, University of Basel, 2007.

$\left[\mathrm{DKP}^{+} 07\right]$ L. Demkowicz, J. Kurtz, D. Pardo, M. Paszynski, W Rachowicz, and S. Zdunek, Computing with hp-adaptive finite elements, Applied Mathematics and Nonlinear Science Series, Chapman \& Hall/CRC, 2007.

[DR06] D. Degerfeldt and T. Rylander, A brick-tetrahedron finite-element interface with stable hybrid explicit-implicit time-stepping for Maxwell's equations, J. Comput. Phys. 220 (2006), no. 1, 383-393.

[FBLV04] C. Fumeaux, D. Baumann, P. Leuchtmann, and R. Vahldieck, A generalized local time-step scheme for efficient FVTD simulations in strongly inhomogeneous meshes, IEEE Trans. Microwave Theory Tech. 52 (2004), no. 3, 1067-1076.

[FLLP05] L. Fezoui, S. Lanteri, S. Lohrengel, and S. Piperno, Convergence and stability of a discontinuous Galerkin time-domain method for the $3 D$ heterogeneous Maxwell equations on unstructuredmeshes, ESAIM: Math. Model. Numer. Anal. 39 (2005), no. 6, 1149-1176.

[Fre03] P. Frey, YAMS: a fully automatic adaptive isotropic surface remeshing procedure, INRIA Research Report No. 4252, 2003.

[GHS91] P.-L. George, F. Hecht, and E. Saltel, Automatic mesh generator with specified boundary, Comput. Methods Appl. Mech. Engrg. 92 (1991), 269-288.

[GLH02] S.G. Garcia, T.W. Lee, and S.C. Hagness, On the accuracy of the ADI-FDTD method, IEEE Trans. Antennas and Wir. Propag. Lett. 1 (2002), 31-34.

[HW02] J.S. Hesthaven and T. Warburton, Nodal high-order methods on unstructured grids. I. Time-domain solution of Maxwell's equations, J. Comput. Phys. 181 (2002), no. 1, 186-221.

[KCGH07] A. Kanevsky, M.A. Carpenter, D. Gottlieb, and J.S. Hesthaven, Application of implicit-explicit high order Runge-Kutta methods to discontinuous Galerkin schemes, J. Comput. Phys. 225 (2007), no. 1753-1781, 2.

[LC87] W. Lorensen and H. Cline, Marching cubes: a high resolution 3D surface construction algorithm, Siggraph 87, vol. 21, 1987, pp. 163-170.

[LF04] J. Lee and B. Fornberg, Some unconditionally stable time stepping methods for the 3d Maxwell's equations, J. Comput. and Appl. Math. 166 (2004), 497-523. 
[MPFC08] E. Montseny, S. Pernet, X. Ferriéres, and G. Cohen, Dissipative terms and local time-stepping improvements in a spatial high order discontinuous Galerkin scheme for the time-domain Maxwell's equations, J. Comput. Phys. 227 (2008), no. $14,6795-6820$.

[MR05] P. Monk and G.R. Richter, A discontinuous Galerkin method for linear symmetric hyperbolic systems in inhomogeneous media, J. Sci. Comput. 22-23 (2005), 443-477.

[Nam00] T. Namiki, 3-D ADI-FDTD method unconditionally stable time-domain algorithm for solving full vector Maxwell's equations, IEEE Trans. Microwave Theory Tech. 48 (2000), no. 10, 1743-1748.

[PFC06] S. Pernet, X. Ferriéres, and G. Cohen, High spatial order finite element method to solve Maxwell's equations in time-domain, IEEE Trans. Antennas and Propag. 53 (2006), no. 9, 2889-2899.

[Pip06] S. Piperno, Symplectic local time stepping in non-dissipative DGTD methods applied to wave propagation problem, ESAIM: Math. Model. Num. Anal. 40 (2006), no. 5, 815-841.

[RB02] T. Rylander and A Bondeson, Stability of explicit-implicit hybrid time-stepping schemes for Maxwell's equations, J. Comput. Phys. 179 (2002), no. 2, 426-438.

[RHGJ03] P. Ratiu, B. Hillen, J. Glaser, and D.P. Jenkins, Medicine Meets Virtual Reality 11 - NextMed: Health Horizon, vol. 11, ch. Visible Human 2.0 - the next generation, pp. 275-281, IOS Press, 2003.

[SSW02] H. Spachmann, R. Schuhmann, and T. Weiland, Higher order explicit time integration schemes for Maxwell's equations, Int. J. Numer. Model.: Electronic Networks, Devices and Fields 15 (2002), no. 4, 419-437.

[TH05] A. Taflove and S.C. Hagness, Computational electrodynamics: the finitedifference time-domain method - 3rd ed., Artech House Publishers, 2005.

[Ver09] J.G. Verwer, Convergence and component splitting for the Crank-Nicolson-LeapFrog integration method, Tech. Report MAS-E0902, Centrum Wiskunde \& Informatica (CWI), 2009.

[Yee66] K.S. Yee, Numerical solution of initial boundary value problems involving Maxwell's equations in isotropic media, IEEE Trans. Antennas and Propag. 14 (1966), no. 3, 302-307.

[ZC01] F. Zheng and S. Chen, Numerical dispersion analysis of the unconditionally stable 3-D ADI-FDTD method, IEEE Trans. Microwave Theory Tech. 49 (2001), no. 5, 1006-1009. 
Unité de recherche INRIA Sophia Antipolis 2004, route des Lucioles - BP 93 - 06902 Sophia Antipolis Cedex (France)

Unité de recherche INRIA Futurs : Parc Club Orsay Université - ZAC des Vignes 4, rue Jacques Monod - 91893 ORSAY Cedex (France) Unité de recherche INRIA Lorraine : LORIA, Technopôle de Nancy-Brabois - Campus scientifique 615, rue du Jardin Botanique - BP 101 - 54602 Villers-lès-Nancy Cedex (France)

Unité de recherche INRIA Rennes : IRISA, Campus universitaire de Beaulieu - 35042 Rennes Cedex (France)

Unité de recherche INRIA Rhône-Alpes : 655, avenue de l'Europe - 38334 Montbonnot Saint-Ismier (France)

Unité de recherche INRIA Rocquencourt : Domaine de Voluceau - Rocquencourt - BP 105 - 78153 Le Chesnay Cedex (France)

INRIA - Domaine de Voluceau - Rocquencourt, BP 105 - 78153 Le Chesnay Cedex (France)

http://www.inria.fr

ISSN 0249-6399 